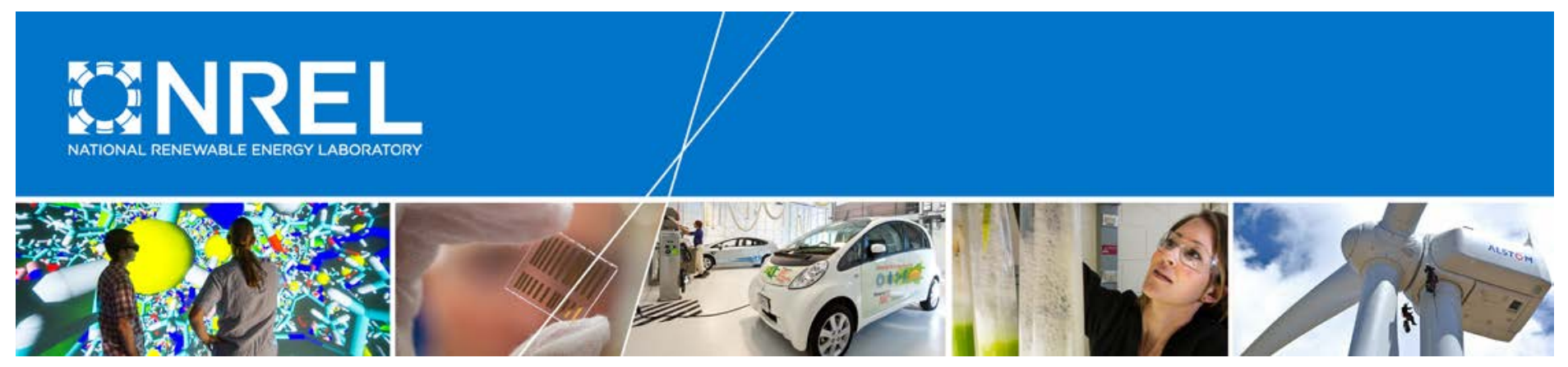

\title{
Advanced Energy System Design (AESD): Technical Manual for the Records API
}

Nicholas Brunhart-Lupo, Brian Bush, Kenny Gruchalla, and Michael Rossol National Renewable Energy Laboratory

NREL is a national laboratory of the U.S. Department of Energy Office of Energy Efficiency \& Renewable Energy Operated by the Alliance for Sustainable Energy, LLC

This report is available at no cost from the National Renewable Energy Laboratory (NREL) at www.nrel.gov/publications.

Technical Report

NREL/TP-6A20-68924

August 2018

Contract No. DE-AC36-08G028308 


\section{Advanced Energy System Design (AESD): Technical Manual for the Records API}

\author{
Nicholas Brunhart-Lupo, Brian Bush, \\ Kenny Gruchalla, and Michael Rossol \\ National Renewable Energy Laboratory
}

\section{Suggested Citation}

Brunhart-Lupo, Nicholas, Brian Bush, Kenny Gruchalla, and Michael Rossol. 2018. Advanced Energy System Design (AESD): Technical Manual for the Records API. Golden, CO: National Renewable Energy Laboratory. NREL/TP-6A20-68924.

https://www.nrel.gov/docs/fy18osti/68924.pdf
National Renewable Energy Laboratory 15013 Denver West Parkway Golden, CO 80401

303-275-3000 • www.nrel.gov
NREL is a national laboratory of the U.S. Department of Energy Office of Energy Efficiency \& Renewable Energy Operated by the Alliance for Sustainable Energy, LLC

This report is available at no cost from the National Renewable Energy Laboratory (NREL) at www.nrel.gov/publications.

\section{Technical Report}

NREL/TP-6A20-68924

August 2018

Contract No. DE-AC36-08GO28308 


\section{NOTICE}

This work was authored by the National Renewable Energy Laboratory, operated by Alliance for Sustainable Energy, LLC, for the U.S. Department of Energy (DOE) under Contract No. DE-AC36-08GO28308. This work was supported by the Laboratory Directed Research and Development (LDRD) Program at NREL. The views expressed in the article do not necessarily represent the views of the DOE or the U.S. Government. The U.S. Government retains and the publisher, by accepting the article for publication, acknowledges that the U.S. Government retains a nonexclusive, paid-up, irrevocable, worldwide license to publish or reproduce the published form of this work, or allow others to do so, for U.S. Government purposes.

This report is available at no cost from the National Renewable Energy Laboratory (NREL) at www.nrel.gov/publications.

U.S. Department of Energy (DOE) reports produced after 1991 and a growing number of pre-1991 documents are available free via www.OSTI.gov. 


\section{Acronyms and Glossary}

ACI

AESD

API

$\mathrm{C}++$

CSV

Chrome

Firefox

Google Protocol Buffers

HTTP

HTTPS

Haskell

IoT

JSON

JavaScript

MySQL

NREL

ODBC

POSIX Epoch

PostgreSQL

Project Haystack

Python

$\mathrm{R}$

REST

Rkt

SQLite3

TSV

URI

UTC

WebSockets

YAML
Application Container Image

Advanced Energy System Design

application programming interface

a programming language

comma-separated-value file

a web browser

a web browser

a serialization specification

Hypertext Transfer Protocol

Hypertext Transfer Protocol Secure

a programming language

Internet of Thinks

JavaScript Object Notation

a programming language

a database server product

National Renewable Energy Laboratory

Open Database Connectivity

seconds since midnight 1 January 1970 UTC

a database server product

a specification for data feeds from the Internet of Thinks (IoT)

a programming language

a programming language

representational state transfer

a container engine (CoreOS 2017b)

a database server product

tab-separate-value file

uniform resource identifier

Coordinated Universal Time

a communication protocol

YAML Ain't Markup Language 


\section{Abstract}

The Records API (application program interface) for Advanced Energy System Design (AESD) enables software that serves multidimensional record-oriented data to interoperate with software that uses such data. In the context of the Records API, multidimensional data records are simply tuples of real numbers, integers, and character strings, where each data value is tagged by a variable name, according to a pre-defined schema, and each record is assigned a unique integer identifier. Conceptually, these records are isomorphic to rows in a relational database, JSON objects, or key-value maps. Records servers might supply static data sets, sensor measurements that periodically update as new telemetry become available, or the results of simulations as the simulations generate new output. Records client software might display or analyze the data, but in the case of simulations, the client requests the creation of new ensembles for specified input parameters. It is also possible to chain records clients and servers together so that a client consuming data from a server might transform that data and serve it to additional clients.

This minimalist API avoids imposing burdensome metadata, or structural or implementation requirements on developers by relying on open source technologies that are readily available for common programming languages. In particular, the API has been designed to place the least possible burden on services that provide data. This document defines the message format for the Records API, a transport mechanism for communicating the data, and the semantics for interpreting it. The message format is specified as Google Protocol Buffers (Google Developers 2017a) and the transport mechanism uses WebSockets (Internet Engineering Task Force 2017). We discuss five major use cases for serving and consuming records data: (1) static data, (2) dynamically augmented data, (3) on-demand simulations, (4) with filters, and (5) with bookmarks. Separate implementations of the API exist in C++, Haskell, JavaScript, Python, and $\mathrm{R}$. 


\section{Table of Contents}

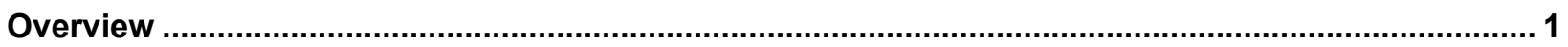

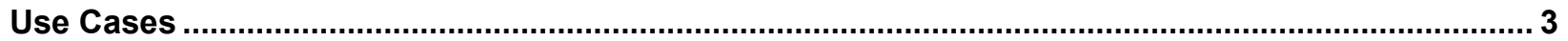

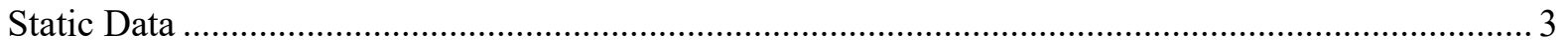

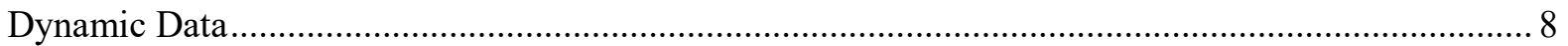

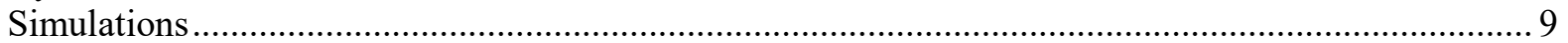

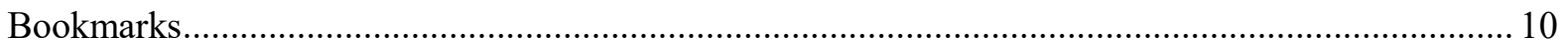

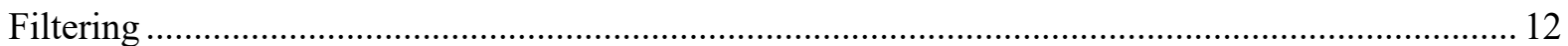

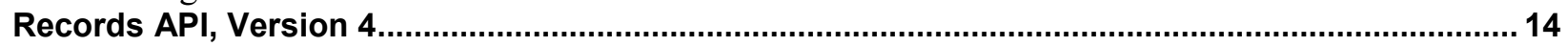

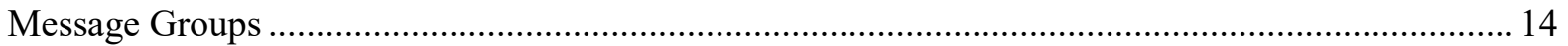

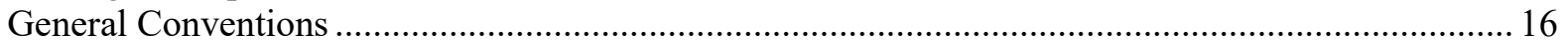

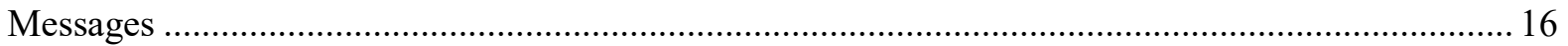

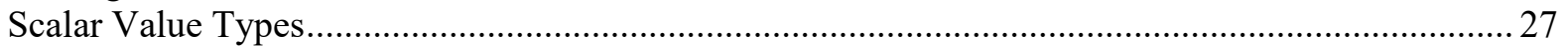

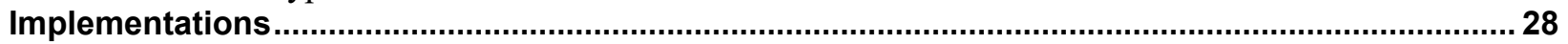

Haskell Client and Server Library and Applications ............................................................. 29

$\mathrm{C}++$ Server and Client .............................................................................................. 33

JavaScript Client Library and Web-Based Browser.............................................................. 33

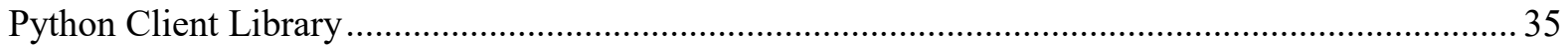

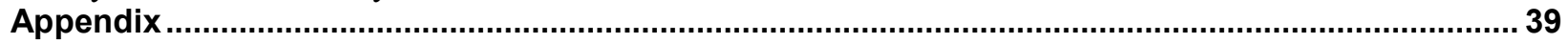

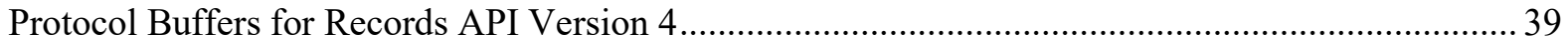

References ............................................................................................................................. 44 


\section{List of Figures}

Containment relationships between protocol buffer messages in the Records API ............................ 2

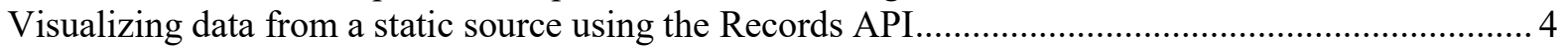

Visualizing data from a dynamic source using the Records API .................................................... 8

Steering and visualizing simulation results using the Records API ................................................ 9

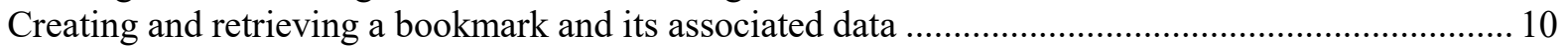

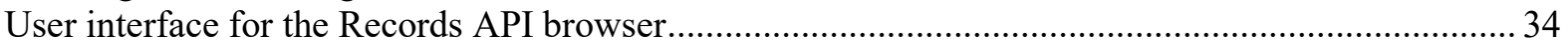

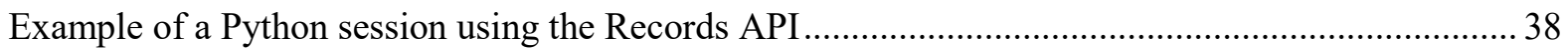

\section{List of Tables}

Correlation between Requests and Responses.............................................................................. 1

Available Client and Server Applications and Libraries for the Records API ................................ 28

Command-Line Arguments for Serving TSV Files................................................................. 31

Parameters for Database Back Ends Serving the Records API .................................................. 32

Command-Line Arguments for Serving Haystack Data Feeds ....................................................... 32

YAML Configuration Parameters for Haystack-Based Records API Servers .................................... 33 


\section{Overview}

Client-server communication in the Records API simply consists of clients sending Request messages to the server and servers asynchronously sending Response messages to the client. The request and response messages hold the specifics of the request or response and the responses are correlated with the requests; however, it is important to note that multiple responses may occur for a single request, as when record data are chunked into multiple response, or that an error response may be sent at any time. The nested messages within Request and Response may in turn contain nested fields and messages providing further details. The table below shows the correspondence between requests and responses, while the figure following that shows the containment relationships between message types.

Correlation between Requests and Responses

\begin{tabular}{ll}
\hline Request Field & Response Field \\
\hline models_metadata & models or error \\
records_data & data or error \\
bookmark_meta & bookmarks or error \\
save_bookmark & bookmarks or error \\
cancel & no response or error \\
work & data or error \\
\hline
\end{tabular}




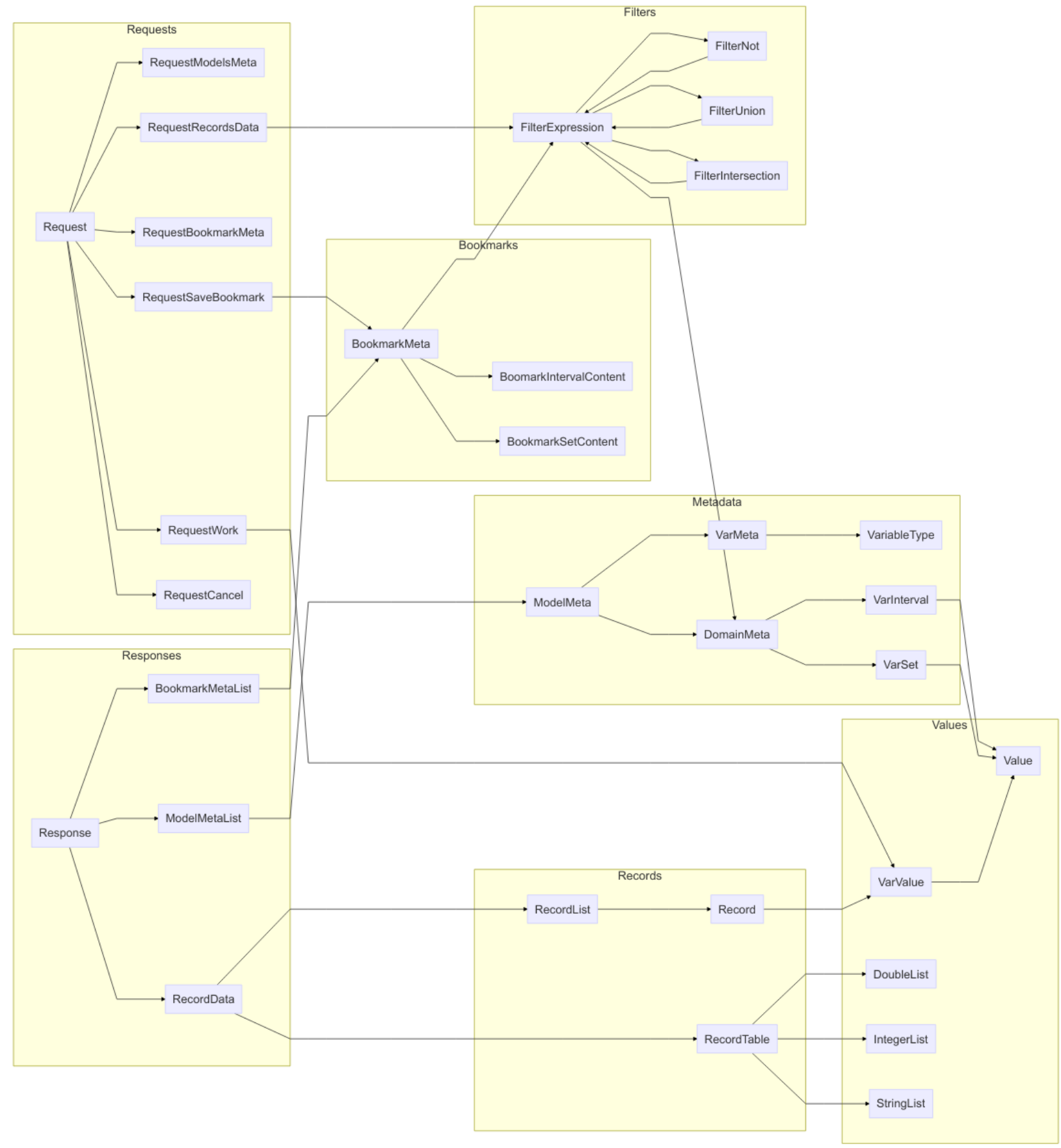

Containment relationships between protocol buffer messages in the Records API 
Metadata messages describe "models," which are just sources of data, and the variables they contain. Data record messages hold the data itself. Data records are simply tuples of real numbers, integers, and character strings, where each data value is tagged by a variable name, according to a pre-defined schema, and each record is assigned a unique integer identifier. Conceptually, these records are isomorphic to rows in a relational database, JSON objects, or key-value maps. For efficiency and compactness, RecordData may be provided in list format or tabular format, with the latter format obtained only when the contents of the table all have the same data type. The data records may be provided in toto or filtered using filter messages so that only certain fields or records are returned. The API contains a small embedded language for filtering via set and value operations. Sets of records may be bookmarked for sharing or later retrieval by (1) enumerating their unique record identifiers, (2) defining a range of unique record identifiers, or (3) specifying a filtering criterion.

Servers that perform computations or simulations can receive input parameters via a RequestWork message that contains those input parameters. After the server has completed its computations, it sends the results as RecordData messages.

In general, the response to a request for data records comes in chunks numbered in sequence, where each chunk has an identifier, chunk_id, and the response specifies the identifier of the next chunk, next_chunk_id. Thus, the chunks form a linked list. The sending of additional chunks can be cancelled using a RequestCancel message. If the subscribe flag is set when making a request, the server will respond indefinitely with additional data as it becomes available, until the subscription is cancelled.

\section{Use Cases}

In this section we outline some standard use cases for the Records API. UML Sequence Diagrams (Fowler 2017) illustrate the flow of messages and the messages themselves are printed in the text format output by the Google protoc tool (Google Developers 2017b).

\section{Static Data}

The retrieval of static data records forms the simplest use case for the Records API. A user chooses a particular data source (a "model" in the parlance of the Records API) and then the data are retrieved and displayed. The visualization client software communicates with a Records server, which in turn accesses the static data. The figure below illustrates the process. 


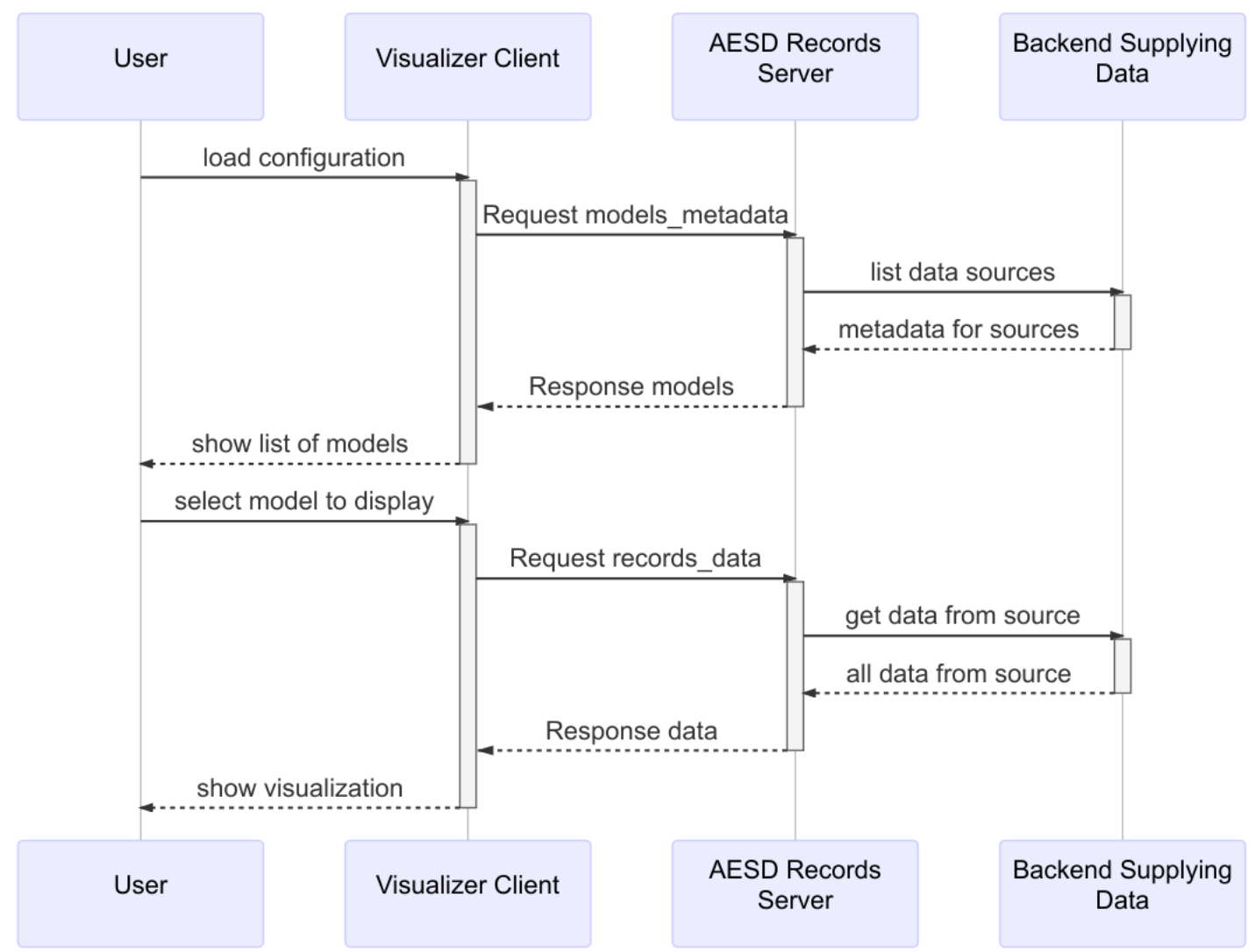

Visualizing data from a static source using the Records API

A Request without model_id specified requests the server to list all models:

version: 4

id: 1

models_metadata \{

\}

The Response from the server provides metadata for all of the models:

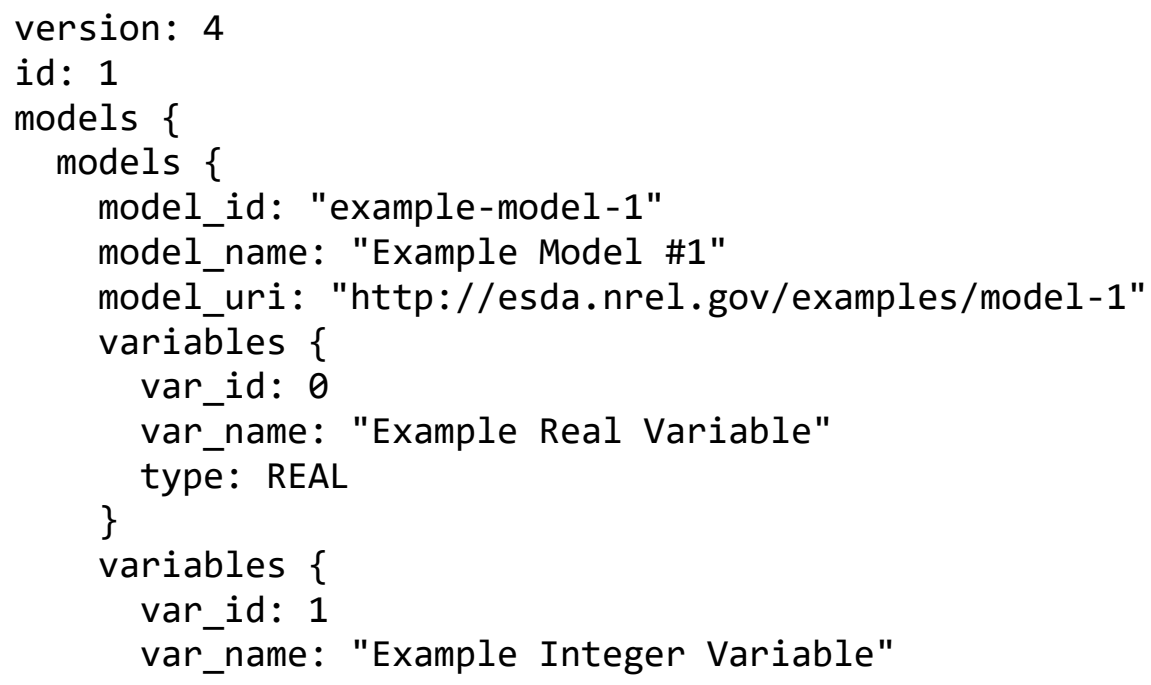




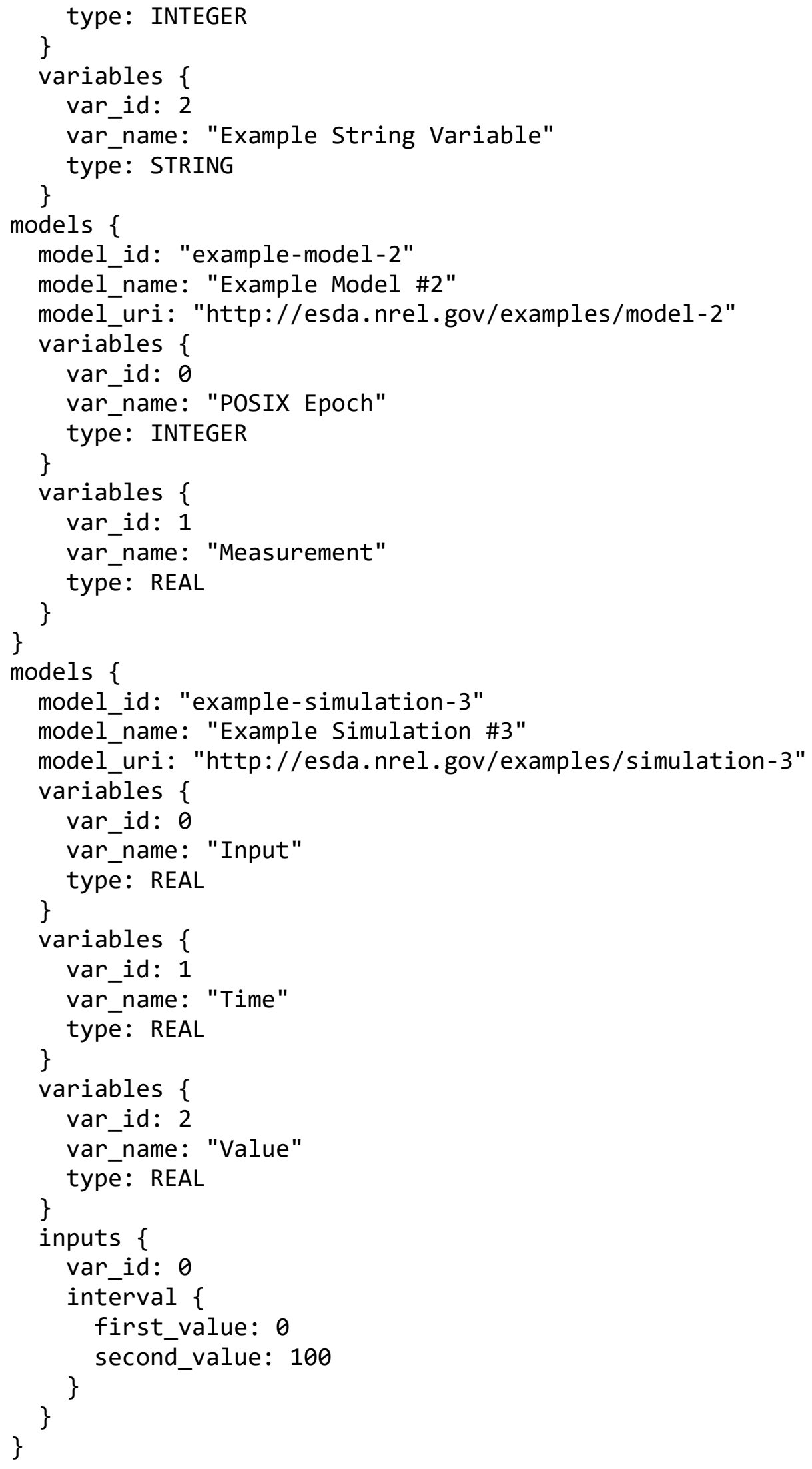


Note that the response above is tagged with the same id as the request; this allows the client to correlate responses with the particular requests it makes. Next, the user might request three records from the first model:

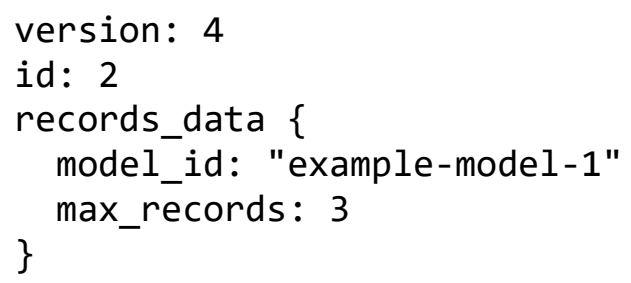

The record data might be returned as two chunks, where the first chunk is

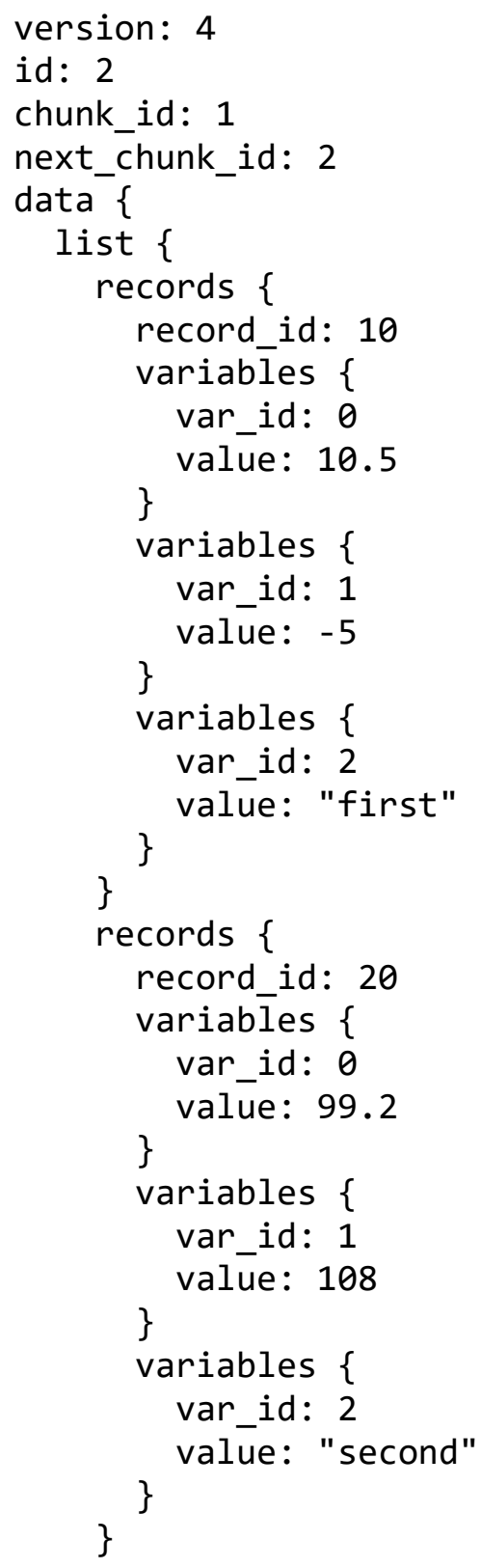




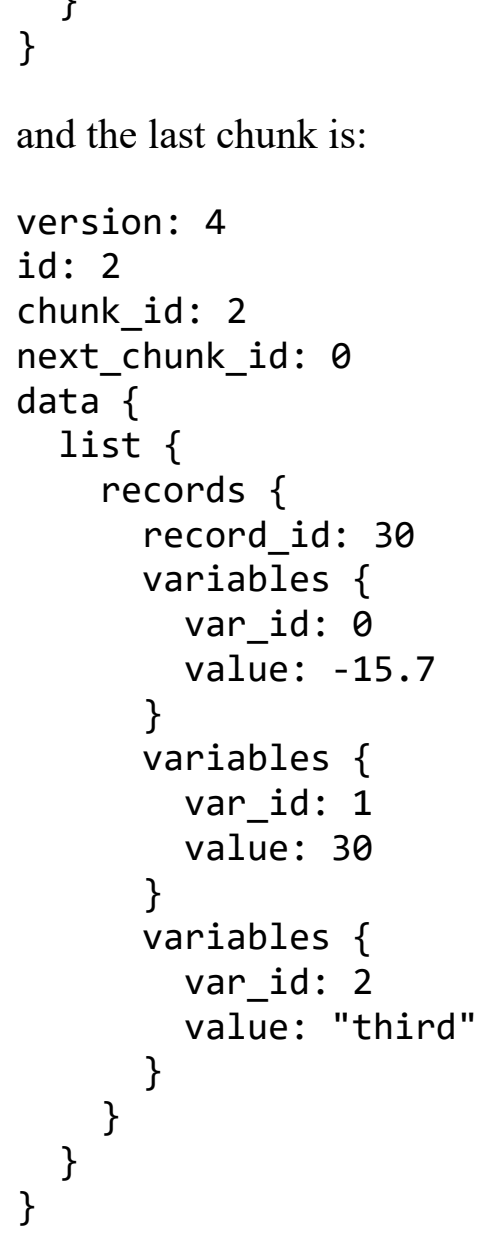




\section{Dynamic Data}

As shown in the following figure, retrieving data from a dynamic source proceeds quite similarly to retrieving data from a static source. The only essential difference is that the server repeatedly sends additional responses containing new data, until a request to cancel is sent.

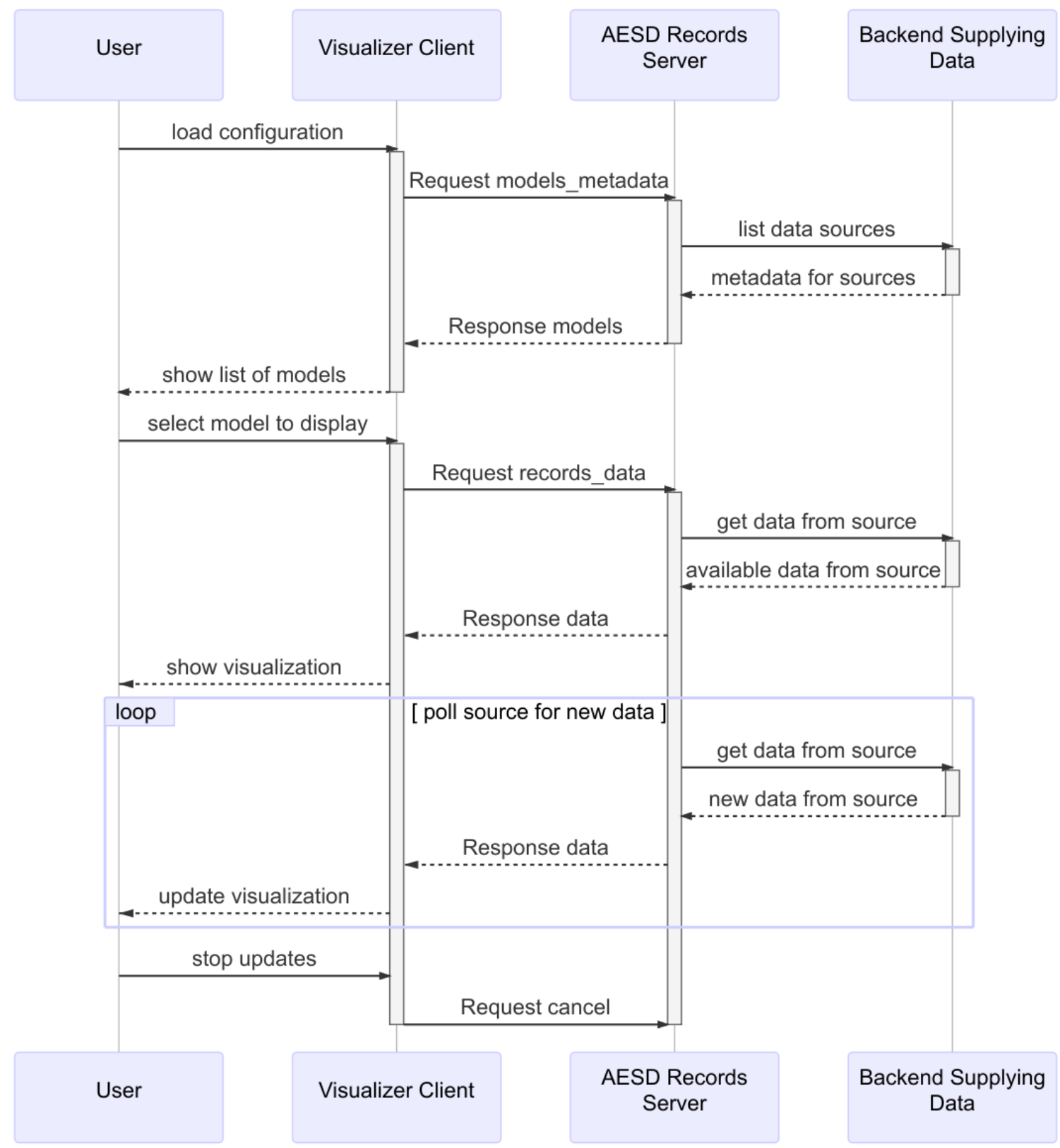

Visualizing data from a dynamic source using the Records API

When requesting dynamic data, it is advisable to set the subscribe flag in the request for data:

version: 4

id: 2

subscribe: true

records_data \{ 
\}

model_id: "example-model-2"

The RequestCancel message is the cancel field Request and must include the id of the request to be cancelled:

version: 4

cancel \{

id: 2

\}

\section{Simulations}

The model Example Simulation \#3 in the Static Data use case is a simulation model, as evidenced by the presence of the inputs field in its metadata. The following figure shows a typical interaction with a simulation-based model via the Records API.

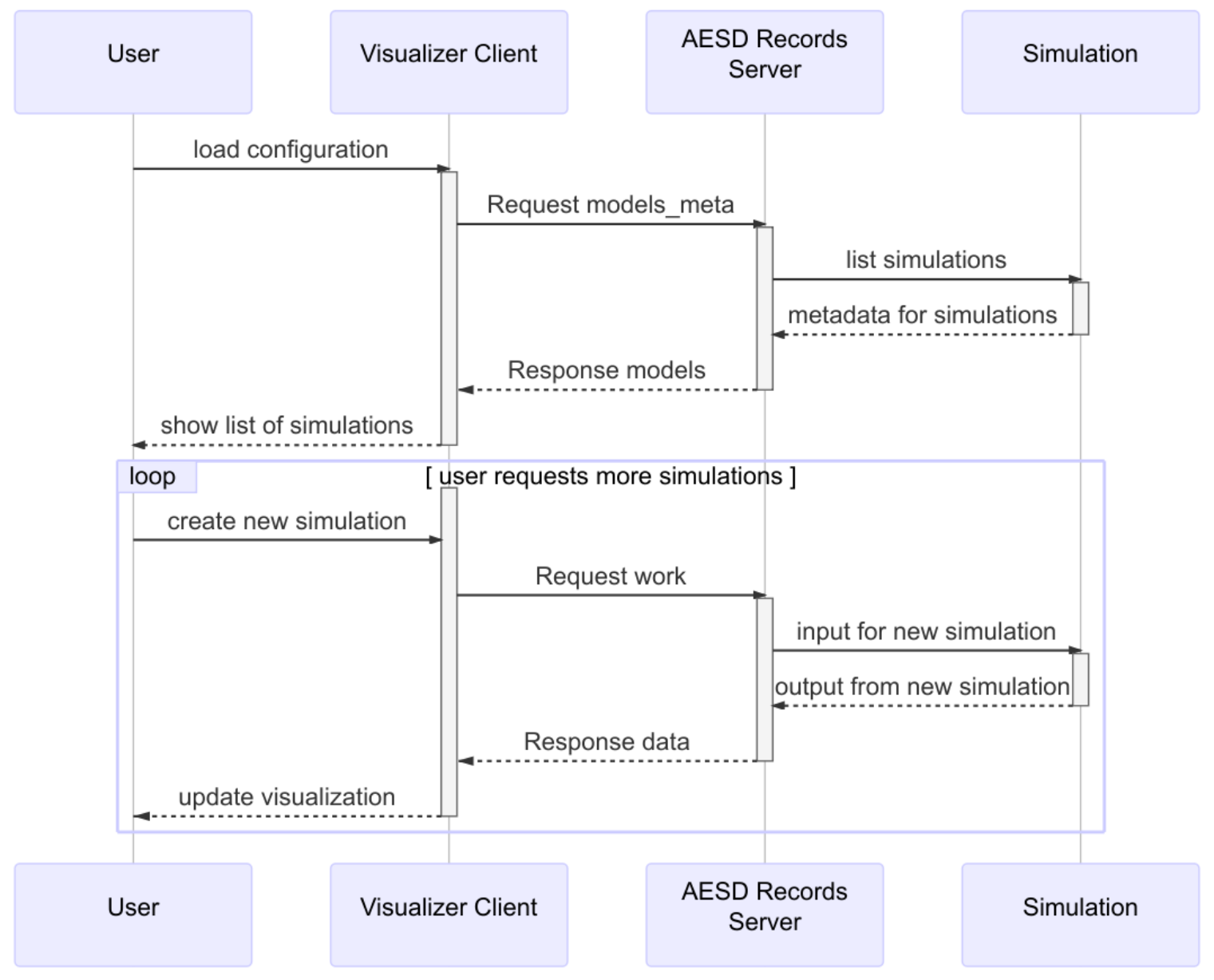

Steering and visualizing simulation results using the Records API 
The RequestWork message, which is contained in the work field of a Request, specifies the input for a simulation to be run:

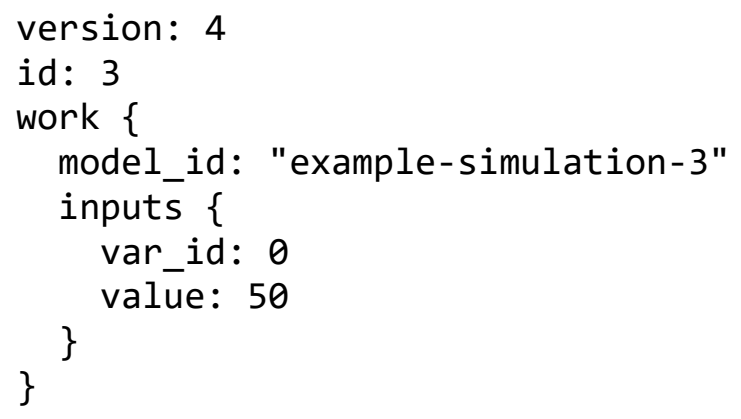

The response to this message will be data for the result of the simulation.

\section{Bookmarks}

Once data from a model is loaded, it may be bookmarked. One simply supplies a description of the data to be bookmarked. Bookmarks can be listed and loaded, as shown in the following figure.

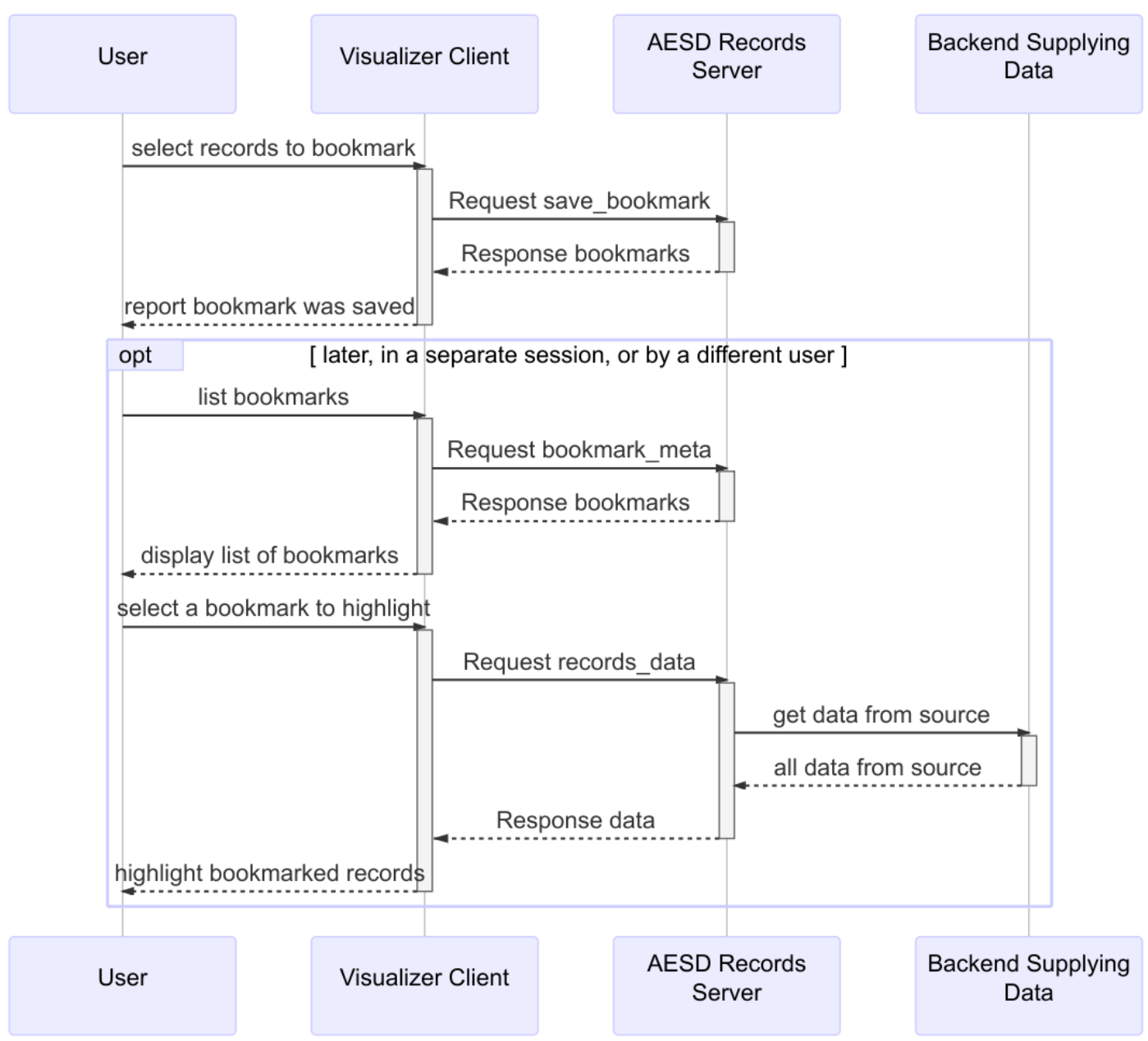

Creating and retrieving a bookmark and its associated data 
To create a bookmark for a specific list of records, simply supply their record identifiers as part of a BookmarkMeta message in the save_bookmark field of Request:

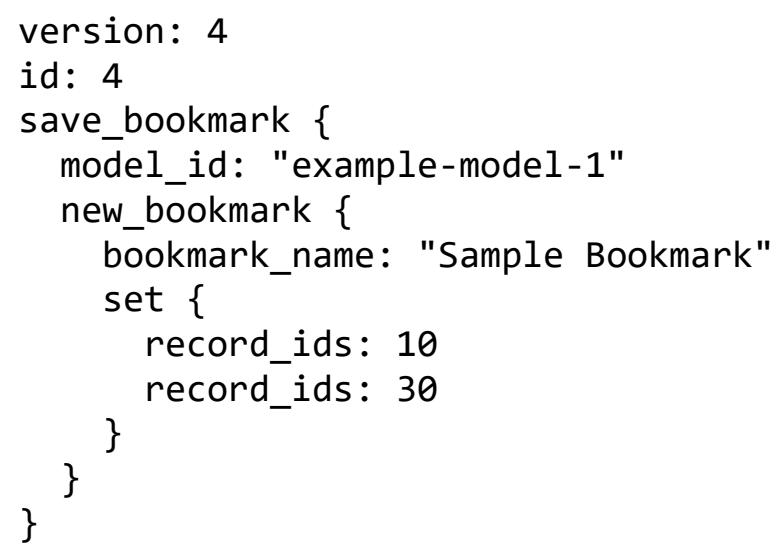

The response will be the same bookmark but with the bookmark_id field added:

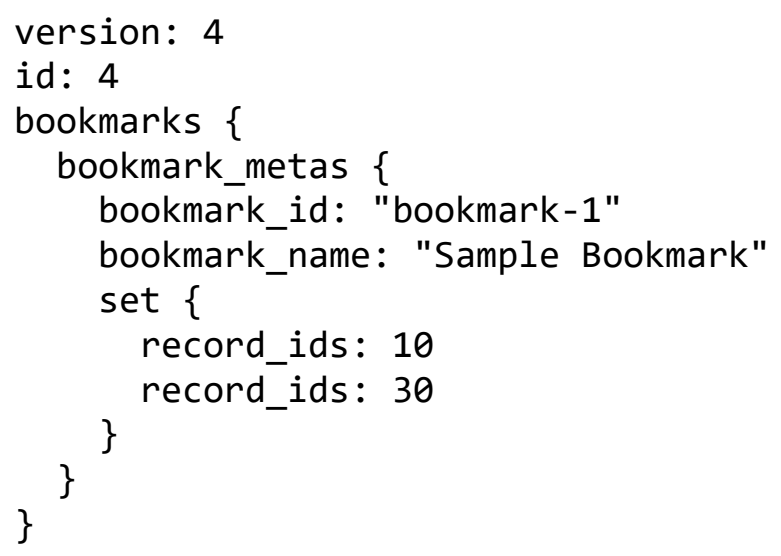

The user, or another user, can retrieve the records corresponding to the bookmark:

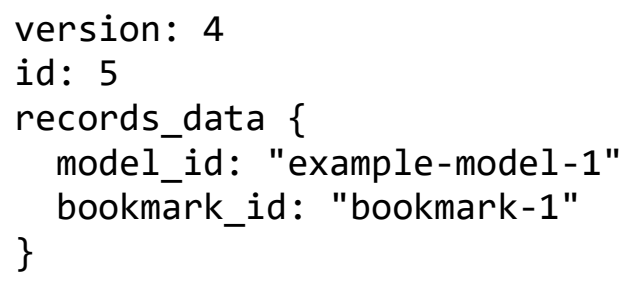

This will return precisely the bookmarked records:

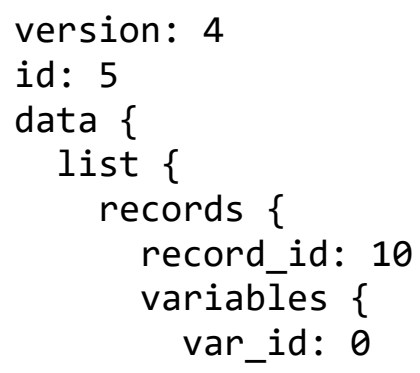




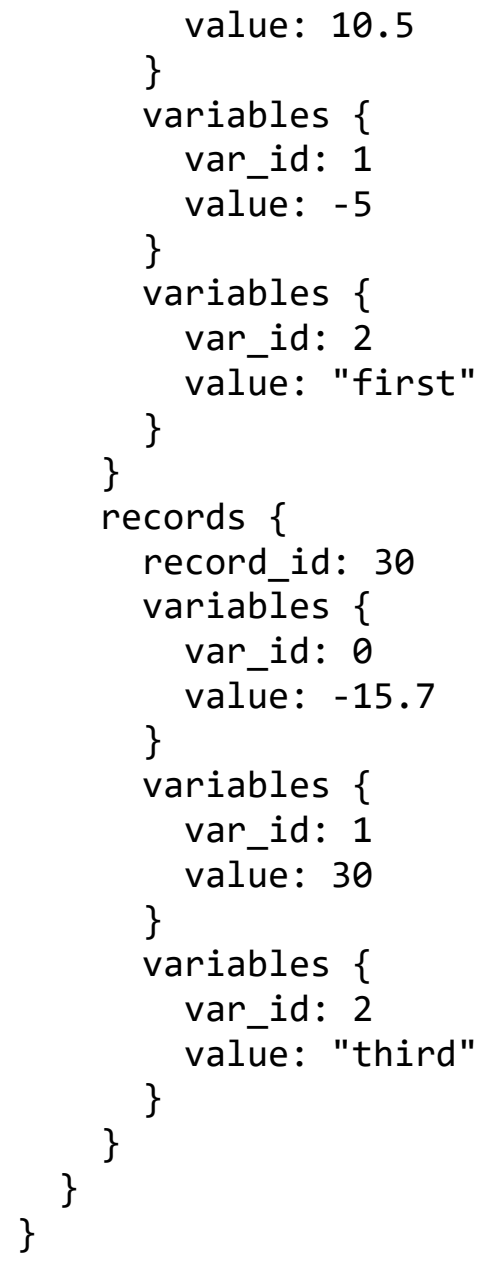

\section{Filtering}

Filtering records can be used to select particular records for retrieval, via the RequestRecordsData message, or in defining bookmarks via the BookmarkMeta message. Filtering of records is accomplished through expressions (FilterExpression), combining values for variables (DomainMeta), and the set operators not, union, and intersection, encoded in the messages FilterNot, FilterUnion, and FitlerIntersection respectively. For example, the expression $x \leq 20$ would be expressed as the following FilterExpression:

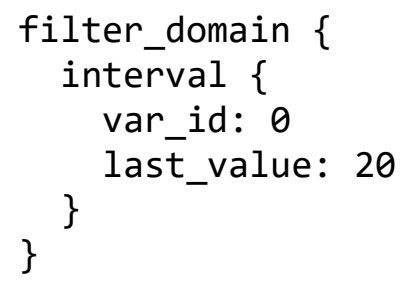

provided that $x$ has var_id $=0$. The expression $(10 \leq x \leq 20) \cup(y \notin\{4,7\})$ would be expressed as

filter_union \{

filter_expressions \{ 


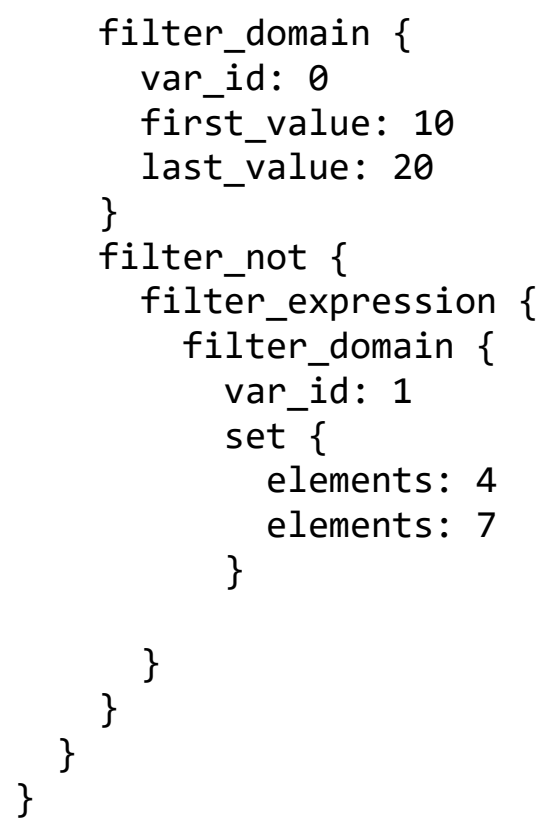

provided that $x$ has var_id $=0$ and $y$ has var_id $=1$. 


\section{Records API, Version 4}

The Records API consists of Google Protobuf 3 (Google Developers 2017b) messages used to request and provide data and metadata for record-oriented information. This section contains the complete specification for Version 4 of the Records API. Clients send Request messages and servers send Response messages, which are typically transported via WebSockets (Internet Engineering Task Force 2017).

\section{Message Groups}

The message types in the Records API are organized into thematic groups below.

\section{Requests and Responses}

Request messages are sent from client to server, and Response messages are sent from server to client. Request messages contain a specific type of request and response messages contain a corresponding specific type of response.

- Request

- RequestModelsMeta

- RequestRecordsData

- RequestWork

- RequestBoomarkMeta

- RequestSaveBookmark

- RequestCancel

- Response

Metadata

Metadata messages describe data sources ("models") and variables.

- ModelMeta

- ModelMetaList

- DomainMeta

- VarMeta

- VariableType

- VarSet 
- VarInterval

\section{Data Records}

Data are represented as either lists of records or tables of them.

- Record

- VarValue

- Value

- RecordData

- RecordList

- RecordTable

\section{Filtering}

Records can be filtered by logical operations on conditions for values of variables in the records.

- FilterExpression

- FilterNot

- FilterIntersection

- FilterUnion

- DomainMeta

\section{Bookmarks}

Bookmarks record particular sets or records or conditions for record data.

- BookmarkMeta

- BookmarkMetaList

- BookmarkIntervalContent

- BookmarkSetContent

Miscellaneous

The following messages wrap data types for the content of records.

- DoubleList

- IntegerList 
- StringList

- OptionalInt32

- OptionalUInt32

- OptionalString

\section{General Conventions}

All fields are technically optional in ProtoBuf 3 , but some fields may be required in each message type in order for the message to be semantically valid. In the following specifications for the messages, fields are annotated as semantically required or semantically optional. Also, the specification notes when field in the protobuf oneof construct are required or mutually exclusive.

Furthermore, one cannot determine whether an optional value has been set if it is just a value, as opposed to a message. That is not true for fields that are messages, where the absence of the field truly indicates the value is absent and is not just a default or unset value. The message Optionalstring, for example, is used in the API to indicate whether a character string value is truly present. Thus RequestModelsMeta has a model_id field that indicates whether the request is for all models, when the field has not been set, or for a specific one, when the field has been set.

Throughout this specification, the following types are used for identifiers: * var_id is int32* model_id is string * record_id is int64

This specification conforms to Protocol Buffers Version 3.

\section{Messages}

\section{BookmarkIntervalContent}

A range of record identifiers can specify the content of a bookmark. Bookmark interval content provides a convenient means to bookmark a contiguous selection of records in a model.

Both fields in this message are optional:

- If neither field is present, the bookmark interval designates all records in the model.

- If only first_record is present, the bookmark interval designates all records starting from that record identifier.

- If only last_record is present, the bookmark interval designates all records ending at that record identifier. For a dynamic model, such a bookmark interval includes all "future" records.

- If both fields are present, the bookmark interval designates all records between the two identifiers, inclusively. 


\begin{tabular}{llll}
\hline Field & Type & Label & Description \\
\hline first_record & int64 & optional & [semantically optional] The identifier for the first record in the interval. \\
last_record & int64 & optional & [semantically optional] The identifier for the last record in the interval. \\
\hline
\end{tabular}

\section{BookmarkMeta}

A bookmark is metadata defining a subset of records in a model.

There are three options for specifying a bookmark:

1. Interval content specifies a range of records in the bookmark.

2. Set content specifies a list of records in the bookmark.

3. A filter expression defines a set of logical conditions for determining whether a record is in the bookmark.

Exactly one of interval, set, or filter must be specified in this message.

\begin{tabular}{|c|c|c|c|}
\hline Field & Type & Label & Description \\
\hline bookmark_id & string & optional & $\begin{array}{l}\text { [semantically optional] When creating a new } \\
\text { bookmark, this field must be empty; the server will } \\
\text { create a unique identifier for the bookmark. This } \\
\text { identifier uniquely identifies the bookmark on the } \\
\text { particular server. }\end{array}$ \\
\hline bookmark_name & string & optional & $\begin{array}{l}\text { [semantically required] a name for the bookmark, } \\
\text { which is useful for displaying the bookmark to users; } \\
\text { this need not be unique, although it is recommended } \\
\text { to be so. }\end{array}$ \\
\hline interval & BookmarklntervalContent & optional & the range of records in the bookmark \\
\hline set & BookmarkSetContent & optional & the list of records in the bookmark \\
\hline filter & FilterExpression & optional & $\begin{array}{l}\text { logical conditions for defining which records are in the } \\
\text { bookmark }\end{array}$ \\
\hline
\end{tabular}

\section{BookmarkMetaList}

Bookmarks may be grouped into lists (sets).

\begin{tabular}{llll}
\hline Field & Type & Label & Description \\
\hline bookmark_metas & BookmarkMeta & repeated & [semantically optional] the bookmarks in the list \\
\hline
\end{tabular}

\section{BookmarkSetContent}

A list (set) of record identifiers can specify the contents of a bookmark. Bookmark-set content provides a convenient means to bookmark a specific selection of non-continuous records in a model.

\begin{tabular}{llll}
\hline Field & Type & Label & Description \\
\hline record_ids & int64 & repeated & $\begin{array}{l}\text { [semantically optional] the list of record identifiers in } \\
\text { the set }\end{array}$ \\
\hline
\end{tabular}




\section{DomainMeta}

The domain (set of valid values) for a variable.

There are two options for specifying a domain:

1. An interval specifies a range of values in the domain.

2. A set specifies a list of values in the domain.

Exactly one of interval or set must be specified in the message.

\begin{tabular}{|c|c|c|c|}
\hline Field & Type & Label & Description \\
\hline var_id & int32 & optional & [semantically required] \\
\hline interval & $\begin{array}{l}\text { Varlnter } \\
\text { val }\end{array}$ & optional & the interval of values in the domain \\
\hline set & VarSet & optional & the list of values in the domain \\
\hline
\end{tabular}

\section{DoubleList}

A list of real numbers.

\begin{tabular}{llll}
\hline Field & Type & Label & Description \\
\hline values & double & repeated & [semantically required] the real numbers \\
\hline
\end{tabular}

\section{FilterExpression}

A filtering expression is a composition of logical conditions on a record. It can be used to filter records. There are four options for specifying a filter expression:

1. The logical negation of another filtering expression

2. The set union of multiple filtering expressions

3. The set intersection of multiple filtering expressions

4. Particular values of variables in a record.

Exactly one of filter_not, filter_union, filter_intersection, or filter_domain must be specified in this message.

\begin{tabular}{llll}
\hline Field & Type & Label & Description \\
\hline filter_not & FilterNot & optional & logical negation of an expression \\
filter_union & FilterUnion & optional & set union of expressions \\
filter_intersection & Filterlntersection & optional & set intersection of expressions \\
filter_domain & DomainMeta & optional & particular values of variables \\
\hline
\end{tabular}




\section{Filterlntersection}

Set intersection of filtering expressions. A record satisfies this expression if it satisfies all filter_expressions.

\begin{tabular}{llll}
\hline Field & Type & Label & Description \\
\hline filter_expressions & FilterExpression & repeated & [semantically required] the expressions to be intersected \\
\hline
\end{tabular}

\section{FilterNot}

Logically negate a filtering expression. A record satisfies this expression if it does not satisfy filter_expression.

\begin{tabular}{llll}
\hline Field & Type & Label & Description \\
\hline filter_expression & FilterExpression & optional & [semantically required] the expression to be negated \\
\hline
\end{tabular}

\section{FilterUnion}

Set union of filtering expressions. A record satisfies this expression if it satisfies any of filter_expressions.

\begin{tabular}{llll}
\hline Field & Type & Label & Description \\
\hline filter_expressions & - & repeated & [semantically required] the expressions to be "unioned" \\
\hline
\end{tabular}

\section{IntegerList}

A list of integers.

\begin{tabular}{llll}
\hline Field & Type & Label & Description \\
\hline values & sint64 & repeated & [semantically required] The integers \\
\hline
\end{tabular}

\section{ModelMeta}

Metadata for a model.

\begin{tabular}{llll}
\hline Field & Type & Label & Description \\
\hline model_id & string & optional & $\begin{array}{l}\text { [semantically required] the unique identifier for the model on the } \\
\text { particular server } \\
\text { model_name }\end{array}$ \\
string & optional & $\begin{array}{l}\text { [semantically required] a name for the model, useful for display the } \\
\text { model to users; this need not be unique, although it is recommended to } \\
\text { be so. }\end{array}$ \\
model_uri & string & optional & $\begin{array}{l}\text { [semantically required] the unique URI for the model; additional } \\
\text { metadata may be obtained by dereferencing that URI. }\end{array}$ \\
variables & VarMeta & repeated & [semantically required] metadata for the variables \\
inputs & DomainMeta & repeated & [semantically optional] metadata for input values to the model, if any \\
\hline
\end{tabular}

\section{ModelMetaList}

A list of metadata for models.

\begin{tabular}{llll}
\hline Field & Type & Label & Description \\
\hline models & ModelMeta & repeated & [semantically optional] the metadata for the models \\
\hline
\end{tabular}




\section{Optionallnt32}

Wrapper for an optional signed integer.

\begin{tabular}{llll}
\hline Field & Type & Label & Description \\
\hline value & int32 & optional & [semantically required] the signed integer value \\
\hline
\end{tabular}

\section{OptionalString}

Wrapper for an optional string.

\begin{tabular}{llll}
\hline Field & Type & Label & Description \\
\hline value & string & optional & [semantically required] the character string value \\
\hline
\end{tabular}

\section{OptionalUInt32}

Wrapper for an optional unsigned integer.

\begin{tabular}{llll}
\hline Field & Type & Label & Description \\
\hline value & uint32 & optional & [semantically required] the unsigned integer value \\
\hline
\end{tabular}

\section{Record}

A record is a list of variables and their associated values.

\begin{tabular}{llll}
\hline Field & Type & Label & Description \\
\hline record_id & int64 & optional & [semantically required] a unique identifier for the record \\
variables & VarValue & repeated & [semantically optional] the values for variables in the record \\
\hline
\end{tabular}




\section{RecordData}

A collection of records.

There are two options for specifying record data:

1. A list specifies a heterogeneously typed list.

2. A table specifies a homogeneously typed table.

Exactly one of List or table must be present in the message.

\begin{tabular}{llll}
\hline Field & Type & Label & Description \\
\hline list & RecordList & optional & a heterogeneously typed list of records \\
table & RecordTable & optional & a homogeneously typed table of records \\
\hline
\end{tabular}

\section{RecordList}

A list of records. The list is heterogeneous in the sense that each variable may have a different type.

\begin{tabular}{llll}
\hline Field & Type & Label & Description \\
\hline records & Record & $\begin{array}{l}\text { repeate } \\
\text { d }\end{array}$ & [semantically optional] The list of records. \\
& & & \\
\hline
\end{tabular}

\section{RecordTable}

A homogeneously typed table of records, where each variable has each type, with a row for each record and a column for each variable.

This message represents the following table:

\begin{tabular}{lllll}
\hline Record Identifier & var_id[0] & var_id[1] & $\ldots$ & var_id[N] \\
\hline rec_id[0] & list[0][0] & list[0][1] & $\ldots$ & list[0][N] \\
rec_id[1] & list[1][0] & list[1][1] & $\ldots$ & list[1][N] \\
$\ldots$ & $\ldots$ & $\ldots$ & $\ldots$ & $\ldots$ \\
rec_id[M] & list[M][0] & list[M][1] & $\ldots$ & list[M][N] \\
\hline
\end{tabular}


The underlying list is a single array, addressable using the following row-major index formula list[row][var] $=$ array[var $+N Y *$ row $]$ where $N X=$ length of rec_ids and NY $=$ length of var_ids.

Exacly one of reals, integers, or strings must be specified in the message.

\begin{tabular}{llll}
\hline Field & Type & Label & Description \\
\hline var_ids & int32 & repeated & [semantically required] the identifiers of the variables (columns) in the table \\
rec_ids & int64 & repeated & [semantically required] the identifiers of the records (rows) in the table \\
reals & DoubleList & optional & the real numbers comprising the values of the variables, in row-major order \\
integers & IntegerList & optional & the integers comprising the values of the variables, in row-major order \\
strings & StringList & optional & the character strings comprising the values of the variables, in row-major order \\
\hline
\end{tabular}

Request

A request. There are six types of requests:

\begin{tabular}{ll}
\hline Request & Response \\
\hline Metadata for model(s) & ModelMetaList \\
Data records & RecordData \\
Metadata for bookmark(s) & BookmarkMetaList \\
Saving a bookmark & BookmarkMetaList \\
Canceling a previous request & $\mathrm{n} / \mathrm{a}$ \\
New work, such as a simulation & RecordData \\
\hline
\end{tabular}

Exactly one of models_metadata, records_data, bookmark_meta, save_bookmark, cancel, or work must be specified in the message.

\begin{tabular}{|c|c|c|c|}
\hline Field & Type & Label & Description \\
\hline version & uint32 & optional & $\begin{array}{l}\text { [semantically required] the version number for the API; } \\
\text { this must be the number four. }\end{array}$ \\
\hline id & OptionalUInt32 & optional & $\begin{array}{l}\text { [semantically optional, but recommended] an identifier } \\
\text { that will be used to tag responses, so that responses can } \\
\text { be correlated with requests }\end{array}$ \\
\hline subscribe & bool & optional & $\begin{array}{l}\text { [semantically optional] whether to continue receiving } \\
\text { responses indefinitely, as new records become } \\
\text { available; this is useful, for example, when a sensor is } \\
\text { reporting measurements periodically or when simulations } \\
\text { are reporting a series or results. Use RequestCancel to } \\
\text { end the subscription. }\end{array}$ \\
\hline models_metadata & RequestModelsMeta & optional & request metadata for model(s) \\
\hline records_data & RequestRecordsData & optional & request data records \\
\hline bookmark_meta & RequestBookmarkMeta & optional & request metadata for bookmark(s) \\
\hline save_bookmark & RequestSaveBookmark & optional & request save a new bookmark or update an existing one \\
\hline cancel & RequestCancel & optional & request to cancel a previous request) \\
\hline work & RequestWork & optional & request work (e.g., simulation results) \\
\hline
\end{tabular}




\section{RequestBookmarkMeta}

A request for one or more bookmarks for a model.

The response to this request is BookmarkMetaList

\begin{tabular}{|c|c|c|c|}
\hline Field & Type & Label & Description \\
\hline model_id & string & optional & $\begin{array}{l}\text { [semantically required] which model for which to list } \\
\text { bookmarks }\end{array}$ \\
\hline bookmark_id & OptionalString & optional & $\begin{array}{l}\text { [semantically optional] If empty, list all bookmarks for the } \\
\text { model. Otherwise, list just the bookmark metadata for this } \\
\text { specific bookmark identifier. }\end{array}$ \\
\hline
\end{tabular}

\section{RequestCancel}

Cancel a previous request.

\begin{tabular}{llll}
\hline Field & Type & Label & Description \\
\hline id & OptionalUInt32 & optional & [semantically required] which request to cancel \\
\hline
\end{tabular}

\section{RequestModelsMeta}

A request for metadata about model(s).

The response to this request is ModelMetaList.

\begin{tabular}{|c|c|c|c|}
\hline Field & Type & Label & Description \\
\hline model_id & OptionalString & optional & $\begin{array}{l}\text { [semantically optional] If absent, the request is for } \\
\text { metadata for all models. Otherwise, the request is for the } \\
\text { specifically identified model. }\end{array}$ \\
\hline
\end{tabular}

\section{RequestRecordsData}

Request record data for a model.

There are three options for requesting record data:

1. Request all records.

2. Request records in a bookmark.

3. Filter records according to a criterion.

The response to this request is RecordData. 
No more than one of bookmark_id or expression may be present in the message.

\begin{tabular}{llll}
\hline Field & Type & Label & Description \\
\hline $\begin{array}{l}\text { model_id } \\
\text { max_records }\end{array}$ & string & uint64 & optional \\
& optional & $\begin{array}{l}\text { [semantically required] the identifier for the model } \\
\text { [semantically optional] If specified, this is the maximum } \\
\text { number of records to return. Otherwise, all records are } \\
\text { returned, although they may be returned as multiple } \\
\text { responses, each with a chunk of records. }\end{array}$ \\
var_ids & int32 & repeated & $\begin{array}{l}\text { [semantically optional] which variables to include in the } \\
\text { response; if this is not specified, all variables will be } \\
\text { included. }\end{array}$ \\
\hline bookmark_id & string & optional & $\begin{array}{l}\text { [semantically optional] Only respond with records in a } \\
\text { specified bookmark. } \\
\text { [semantically optional] Only respond with records }\end{array}$ \\
\hline
\end{tabular}

\section{RequestSaveBookmark}

A request to create or update a bookmark.

The response to this request is BookmarkMetaList.

\begin{tabular}{llll}
\hline Field & Type & Label & Description \\
\hline model_id & string & optional & $\begin{array}{l}\text { [semantically required] which model for which to save the } \\
\text { bookmark }\end{array}$ \\
new_bookmark & BookmarkMeta & optional & $\begin{array}{l}\text { [semantically optional] If empty, create a new bookmark. } \\
\text { (In which case, leave the bookmark_id empty, so that } \\
\text { the server will create a unique identifier for the new } \\
\text { bookmark.) Otherwise, update an existing bookmark. }\end{array}$ \\
\hline
\end{tabular}

\section{RequestWork}

Request that the server compute new records based on input values.

The response to this request is RecordData.

\begin{tabular}{|c|c|c|c|}
\hline Field & Type & Label & Description \\
\hline model_id & string & optional & [semantically required] the identifier for the model \\
\hline inputs & VarValue & repeated & $\begin{array}{l}\text { [semantically optional] which input variables to set to } \\
\text { which values }\end{array}$ \\
\hline
\end{tabular}




\section{Response}

A response to a request.

Note that a server may send multiple responses to a single request, expressed as a linked list of chunks. It is strongly recommended that servers chunk by record_id so that each record is kept intact. A chunk may be empty.

\begin{tabular}{|c|c|c|c|}
\hline Field & Type & Label & Description \\
\hline version & uint32 & optional & $\begin{array}{l}\text { [semantically required] the version number for the API; } \\
\text { this must be the number four. }\end{array}$ \\
\hline id & OptionalUInt32 & optional & $\begin{array}{l}\text { [semantically optional] a response without an identifier is a } \\
\text { notification; otherwise, the response identifier matches the } \\
\text { response identifier for the original request. }\end{array}$ \\
\hline chunk_id & int32 & optional & $\begin{array}{l}\text { [semantically optional, but recommended] the identifier for } \\
\text { this chunk; it is recommended that chunks are numbered } \\
\text { sequentially starting beginning with the number one. }\end{array}$ \\
\hline next_chunk_id & int32 & optional & $\begin{array}{l}\text { [semantically optional] the identifier of the next chunk, or } \\
\text { zero if this is the last chunk }\end{array}$ \\
\hline error & string & optional & an error message \\
\hline models & ModelMetaList & optional & a list of model metadata \\
\hline data & RecordData & optional & a list of record data \\
\hline bookmarks & BookmarkMetaList & optional & a list of bookmark metadata \\
\hline
\end{tabular}

StringList

A list of character strings.

\begin{tabular}{llll}
\hline Field & Type & Label & Description \\
\hline values & string & repeated & [semantically required] the character strings \\
\hline
\end{tabular}

\section{Value}

Value that may be a real number, an integer, or a character string

Exactly one of real_value, integer_value, or string_value must be specified in this message.

\begin{tabular}{llll}
\hline Field & Type & Label & Description \\
\hline real_value & double & optional & the real number \\
integer_value & int64 & optional & the integer \\
string_value & string & optional & the character string \\
\hline
\end{tabular}




\section{Varlnterval}

A range of values of a variable.

Both fields in this message are optional:

- If neither field is present, the interval designates all values in the domain.

- If only first_value is present, the interval designates all values starting from that value.

- If only last_value is present, the bookmark interval designates all values ending at that value.

- If both fields are present, the interval designates all values between the two values, inclusive.

\begin{tabular}{llll}
\hline Field & Type & Label & Description \\
\hline first_value & Value & optional & [semantically optional] the first value in the interval \\
last_value & Value & optional & [semantically optional] the last value in the interval \\
\hline
\end{tabular}

\section{VarMeta}

Metadata for a variable.

\begin{tabular}{|c|c|c|c|}
\hline Field & Type & Label & Description \\
\hline var_id & int32 & optional & $\begin{array}{l}\text { [semantically required] an integer identifying } \\
\text { the variable }\end{array}$ \\
\hline var_name & string & optional & [semantically required] the name of the variable \\
\hline units & string & optional & $\begin{array}{l}\text { [semantically optional] the name of the unit of } \\
\text { measure for values of the variable }\end{array}$ \\
\hline si & $\sin 132$ & repeated & $\begin{array}{l}\text { [semantically optional] the unit of measure } \\
\text { expressed as a list of the exponents for the eight } \\
\text { fundamental SI quantities [meter, kilogram, } \\
\text { second, ampere, kelvin, mole, candela, radian]; } \\
\text { for example, the unit of acceleration } m / \mathrm{s}^{2} \text { would } \\
\text { be express as }[1,0,-2,0,0,0,0, \\
0] \text { because meters has an exponent of positive } \\
\text { one and seconds has an exponent of negative } \\
\text { two. }\end{array}$ \\
\hline scale & double & optional & $\begin{array}{l}\text { [semantically optional] An overall scale relative to } \\
\text { the fundamental SI scale of the unit of measure; } \\
\text { for instance, kilometers would have a scale of } \\
1,000 \text { because the fundamental unit of distance is } \\
\text { meters. }\end{array}$ \\
\hline type & VariableType & optional & $\begin{array}{l}\text { [semantically optional] the data type for values of } \\
\text { the variable; The default type is real number. }\end{array}$ \\
\hline
\end{tabular}

\section{VarSet}

A set of values for a variable.

\begin{tabular}{llll}
\hline Field & Type & Label & Description \\
\hline elements & Value & repeated & [semantically optional] the list of values in the set \\
\hline
\end{tabular}




\section{VarValue}

The value of a variable.

\begin{tabular}{llll}
\hline Field & Type & Label & Description \\
\hline var_id & int32 & optional & $\begin{array}{l}\text { [semantically required] the identifier for the } \\
\text { variable }\end{array}$ \\
value & Value & optional & [semantically required] the value of the variable \\
\hline
\end{tabular}

\section{VariableType}

The data type for a value.

\begin{tabular}{lll}
\hline Name & Number & Description \\
\hline REAL & 0 & a real number \\
INTEGER & 1 & an integer \\
STRING & 2 & a character string \\
\hline
\end{tabular}

\section{Scalar Value Types}

\begin{tabular}{|c|c|c|c|c|}
\hline $\begin{array}{l}\text { proto } \\
\text { Type }\end{array}$ & Notes & $\begin{array}{l}\text { C++ } \\
\text { Type }\end{array}$ & $\begin{array}{l}\text { Java } \\
\text { Type }\end{array}$ & $\begin{array}{l}\text { Python } \\
\text { Type }\end{array}$ \\
\hline double & & double & double & float \\
\hline float & & float & float & float \\
\hline int32 & $\begin{array}{l}\text { Uses variable-length encoding. Inefficient for encoding } \\
\text { negative numbers-if your field is likely to have negative } \\
\text { values, use sint32 instead. }\end{array}$ & int32 & int & int \\
\hline int64 & $\begin{array}{l}\text { Uses variable-length encoding. Inefficient for encoding } \\
\text { negative numbers; if your field is likely to have negative } \\
\text { values, use sint } 64 \text { instead. }\end{array}$ & int64 & long & int/long \\
\hline uint32 & Uses variable-length encoding. & uint32 & int & int/long \\
\hline uint64 & Uses variable-length encoding. & uint64 & long & int/long \\
\hline $\sin 132$ & $\begin{array}{l}\text { Uses variable-length encoding. Signed int value. These more } \\
\text { efficiently encode negative numbers than regular int32s. }\end{array}$ & int32 & int & int \\
\hline $\operatorname{sint64}$ & $\begin{array}{l}\text { Uses variable-length encoding. Signed int value. These more } \\
\text { efficiently encode negative numbers than regular int } 64 \mathrm{~s} \text {. }\end{array}$ & int64 & long & int/long \\
\hline fixed32 & $\begin{array}{l}\text { Always four bytes. More efficient than uint } 32 \text { if values are often } \\
\text { greater than } 2^{\wedge} 28 .\end{array}$ & uint32 & int & int \\
\hline fixed 64 & $\begin{array}{l}\text { Always eight bytes. More efficient than uint } 64 \text { if values are } \\
\text { often greater than } 2^{\wedge} 56 \text {. }\end{array}$ & uint64 & long & int/long \\
\hline sfixed32 & Always four bytes. & int32 & int & int \\
\hline sfixed64 & Always eight bytes. & int64 & long & int/long \\
\hline bool & & bool & Boolean & Boolean \\
\hline string & $\begin{array}{l}\text { A string must always contain UTF-8 encoded or 7-bit ASCII } \\
\text { text. }\end{array}$ & string & String & str/unicode \\
\hline bytes & May contain any arbitrary sequence of bytes. & string & ByteString & str \\
\hline
\end{tabular}




\section{Implementations}

This section provides an overview of the variety of libraries and applications implementing the Records API (see the table below). In particular, pre-built applications are available for serving text-based data sources, database queries, and sensor data feeds. Application Container Images (ACIs) (CoreOS 2017a) of each have been packed for use with the rkt container engine (CoreOS 2017b).

Available Client and Server Applications and Libraries for the Records API

\begin{tabular}{|c|c|c|c|c|c|}
\hline $\begin{array}{l}\text { Client } \\
\text { or } \\
\text { Server } \\
?\end{array}$ & $\begin{array}{l}\text { Library or } \\
\text { Application } \\
?\end{array}$ & $\begin{array}{l}\text { Data } \\
\text { Source }\end{array}$ & $\begin{array}{l}\text { Implementation } \\
\text { Language }\end{array}$ & $\begin{array}{l}\text { Computing } \\
\text { Platforms }\end{array}$ & URL \\
\hline client & $\begin{array}{l}\text { GUI } \\
\text { application }\end{array}$ & any & $\mathrm{C}++$ & $\begin{array}{l}\text { Mac, } \\
\text { Winodws, } \\
\text { Linux }\end{array}$ & https://github.nrel.gov/d-star/cpp-records \\
\hline server & $\begin{array}{l}\text { GUI/CLI } \\
\text { applications }\end{array}$ & CSV files & $\mathrm{C}++$ & $\begin{array}{l}\text { Mac, } \\
\text { Winodws, } \\
\text { Linux }\end{array}$ & https://github.nrel.gov/d-star/cpp-records \\
\hline client & library & any & Haskell & $\begin{array}{l}\text { Mac, } \\
\text { Windows, } \\
\text { Linux }\end{array}$ & https://github.com/NREL/AESD/lib/haskel \\
\hline server & $\begin{array}{l}\text { CLI } \\
\text { application }\end{array}$ & TSV files & Haskell & $\begin{array}{l}\text { Mac, } \\
\text { Windows, } \\
\text { Linux }\end{array}$ & https://github.com/NREL/AESD/lib/haskel \\
\hline server & $\begin{array}{l}\text { CLI } \\
\text { application }\end{array}$ & PostgreSQ & Haskell & $\begin{array}{l}\text { Mac, } \\
\text { Windows, } \\
\text { Linux }\end{array}$ & https://github.com/NREL/AESD/lib/haskel \\
\hline server & $\begin{array}{l}\text { CLI } \\
\text { application }\end{array}$ & MySQL & Haskell & $\begin{array}{l}\text { Mac, } \\
\text { Windows, } \\
\text { Linux }\end{array}$ & https://github.com/NREL/AESD/lib/haskel \\
\hline server & $\begin{array}{l}\text { CLI } \\
\text { application }\end{array}$ & SQLite3 & Haskell & $\begin{array}{l}\text { Mac, } \\
\text { Windows, } \\
\text { Linux }\end{array}$ & https://github.com/NREL/AESD/lib/haskel \\
\hline server & $\begin{array}{l}\text { CLI } \\
\text { application }\end{array}$ & ODBC & Haskell & $\begin{array}{l}\text { Mac, } \\
\text { Windows, } \\
\text { Linux }\end{array}$ & https://github.com/NREL/AESD/lib/haskel \\
\hline server & $\begin{array}{l}\text { CLI } \\
\text { application }\end{array}$ & Haystack & Haskell & $\begin{array}{l}\text { Mac, } \\
\text { Windows, } \\
\text { Linux }\end{array}$ & https://github.com/NREL/AESD/lib/haskel \\
\hline client & $\begin{array}{l}\text { library, web } \\
\text { application }\end{array}$ & any & JavaScript & $\begin{array}{l}\text { Chrome, } \\
\text { Firefox }\end{array}$ & $\begin{array}{l}\text { https://github.com/NREL/AESD/lib/javasc } \\
\text { ript }\end{array}$ \\
\hline client & library & any & Python & any & https://github.com/NREL/AESD/lib/python \\
\hline client & library & any & $\mathrm{R}$ & any & https://github.nrel.gov/d-star/r-records \\
\hline
\end{tabular}




\section{Haskell Client and Server Library and Applications}

Both client and server applications in Haskell are available for the Records API.

Full documentation resides at https://github.com/NREL/AESD/lib/haskell.

\section{Client Library}

The client library described below provides the basic functions for interacting with any

Records API server.

Types

data State

State information for a client.

Entry Point

clientMain

Run a client.

\begin{tabular}{|c|c|}
\hline Argument Type & Description \\
\hline :: String & the WebSocket host address \\
\hline$->$ Int & the WebSocket port number \\
\hline -> String & the WebSocket path \\
\hline$->($ State $->$ IO ()$)$ & customize the client \\
\hline$->$ IO () & action for running the client \\
\hline
\end{tabular}

Close a client.

\begin{tabular}{ll}
\hline Argument Type & Description \\
\hline$::$ State & the state of the client \\
$->$ IO ( ) & action for closing the client \\
\hline
\end{tabular}

Server Requests

fetchModels

Fetch model metadata.

\begin{tabular}{ll}
\hline Argument Type & Description \\
\hline$::$ State & the state of the client \\
$->$ IO (Either String [ModelMeta]) & action returning either an error or the models \\
\hline
\end{tabular}




\section{fetchRecords}

Fetch records from the server.

\begin{tabular}{ll}
\hline Argument Type & Description \\
\hline$::$ State & the state of the client \\
$->$ ModelIdentifier & the model identifier \\
$->$ Maybe Int & the maximum number of records to request \\
$->$ IO (Either String [RecordContent]) & action returning either an error or the records \\
\hline
\end{tabular}

\section{fetchBookmarks}

Fetch bookmark(s).

\begin{tabular}{ll}
\hline Argument Type & Description \\
\hline$::$ State & the state of the client \\
-> ModelIdentifier & the model identifier \\
$->$ Maybe BookmarkIdentifier & the bookmark identifier, or all bookmarks \\
$->$ IO (Either String [BookmarkMeta]) & action returning either an error or the bookmark(s) \\
\hline
\end{tabular}

storeBookmark

Save a bookmark.

\begin{tabular}{ll}
\hline Argument Type & Description \\
\hline$::$ State & the state of the client \\
$->$ ModelIdentifier & the model identifier \\
\hline$->$ BookmarkMeta & the bookmark metadata \\
$->$ IO (Either String BookmarkMeta) & action returning either an error or the bookmark \\
\hline
\end{tabular}

\section{Server Library}

The server library provides two options for implementing a Records API server. The AESD. Records.Server module provides a main entry point serverMain, a type class ModelManager, and a monad ServiceM that implement a skeletal server, which handles all of the WebSocket communication and Protocol Buffer serialization; an implementer need only create an instance of ModelManager. Furthermore, the AESD. Records.Server. Manager module provides such an instance InMemoryManager of the type class ModelManger to handle inmemory caching of data and on-disk persistence of bookmarks; here, an implementer just calls the function makeInMemoryManager and provides several functions that retrieve content: 
makeInMemoryManager

Construct an in-memory model manager.

\begin{tabular}{ll}
\hline Argument Type & Description \\
\hline$::$ Maybe FilePath & the name of the journal file \\
$->$ a & the initial state \\
$->(a->$ IO ([ModelMeta $]$, a $))$ & $\begin{array}{l}\text { list models in an action modifying the } \\
\text { state }\end{array}$ \\
$->(a->$ ModelMeta $->$ IO $([$ RecordContent $], a))$ & $\begin{array}{l}\text { load record data in an action } \\
\text { modifying the state }\end{array}$ \\
$->(a->$ ModelMeta $->[$ VarValue $]->$ IO & $\begin{array}{l}\text { performing work in an action } \\
\text { modifying the state }\end{array}$ \\
$([$ RecordContent $]$, a) & action constructing the manager \\
$->$ IO (InMemoryManager a) &
\end{tabular}

\section{Server Back Ends}

As previously mentioned, prebuilt servers have been implemented for standard types of data sources.

\section{Tab-Separate-Value Files}

Serving tab-separated-value (TSV) files is as simple as placing the TSV files in a directory and starting a server at the command line, with the arguments specified in the table below:

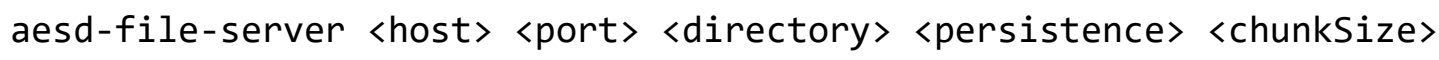

\section{Command-Line Arguments for Serving TSV Files}

\begin{tabular}{ll}
\hline Parameter & Description \\
\hline host & host address to which to bind the service \\
port & port to which to bind the service \\
directory & directory with TSV files to be served \\
persistence & filename for bookmark data \\
chunkSize & number of records return in each chunk \\
\hline
\end{tabular}




\section{Database Queries}

The Records API servers have been implemented for the most common database back ends. Each server takes a single command-line argument specifying a YAML (Oren Ben-Kiki, Clark Evans, Ingy döt Net 2017) configuration file with the parameters in the table below.

Parameters for Database Back Ends Serving the Records API

\begin{tabular}{|c|c|c|c|c|c|}
\hline Parameter & Description & PostgreSQL & MySQL & SQLite3 & ODBC \\
\hline host & $\begin{array}{l}\text { host address to } \\
\text { which to bind the } \\
\text { service }\end{array}$ & required & required & required & required \\
\hline port & $\begin{array}{l}\text { port to which to bind } \\
\text { the service }\end{array}$ & required & required & required & required \\
\hline directory & $\begin{array}{l}\text { directory with queries } \\
\text { to be served }\end{array}$ & required & required & required & required \\
\hline persistence & $\begin{array}{l}\text { filename for } \\
\text { bookmark data }\end{array}$ & optional & optional & optional & optional \\
\hline chunkSize & $\begin{array}{l}\text { number of records } \\
\text { return in each chunk }\end{array}$ & optional & optional & optional & optional \\
\hline database & $\begin{array}{l}\text { database connection } \\
\text { information }\end{array}$ & $\begin{array}{l}\text { required } \\
\text { connection string }\end{array}$ & $\begin{array}{l}\text { required } \\
\text { connection } \\
\text { string }\end{array}$ & $\begin{array}{l}\text { required } \\
\text { filename }\end{array}$ & $\begin{array}{l}\text { required } \\
\text { connection } \\
\text { string }\end{array}$ \\
\hline
\end{tabular}

\section{Haystack Sensor Measurements and the "Internet of Things"}

Furthermore, a server for Project Haystack (Project Haystack 2017) data feeds, typically sensor measurements from devices in the "internet of things," has been implemented. The server takes command-line arguments specified in the table below.

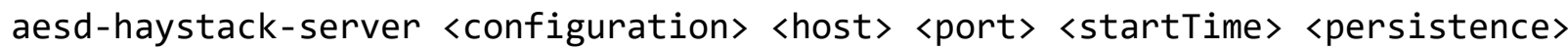
<chunkSize>

\section{Command-Line Arguments for Serving Haystack Data Feeds}

\begin{tabular}{ll}
\hline Parameter & Description \\
\hline $\begin{array}{l}\text { configuration } \\
\text { host }\end{array}$ & YAML configuration file for accessing the Haystack service \\
port & host address to which to bind the service \\
startTime & earliest time to serve, specified in seconds of the POSIX Epoch \\
persistence & filename for bookmark data \\
chunkSize & number of records return in each chunk \\
\hline
\end{tabular}


The parameters in the YAML configuration file like the one below and are described in the following table:

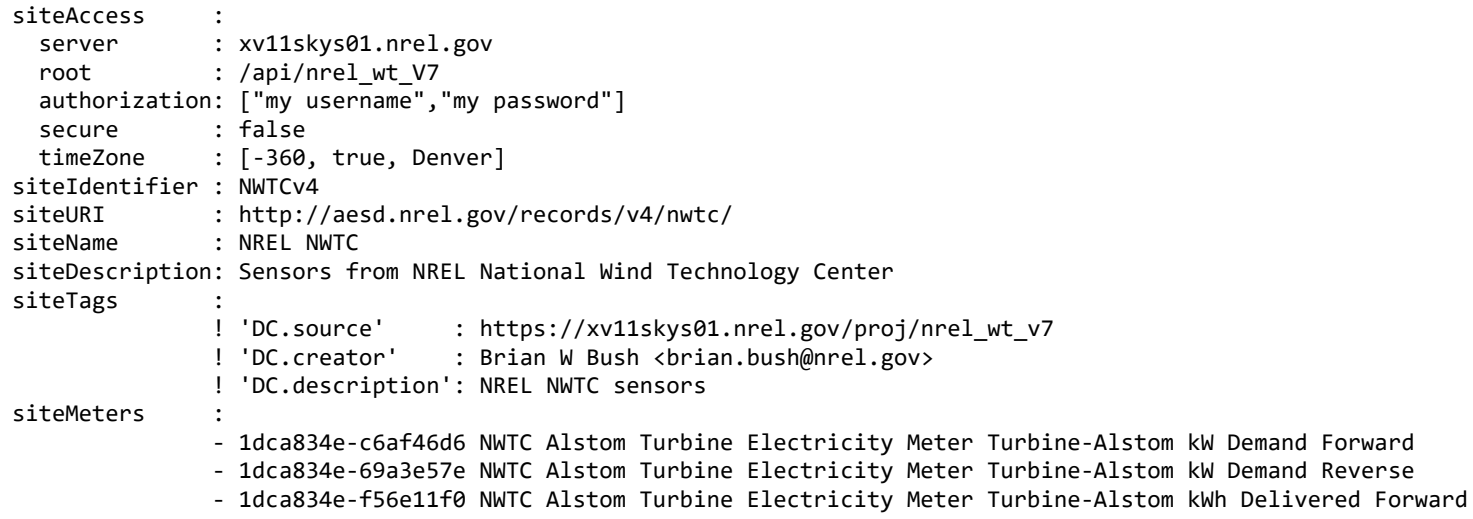

YAML Configuration Parameters for Haystack-Based Records API Servers

\begin{tabular}{|c|c|c|}
\hline Parameter & Description & Required? \\
\hline server & hostname and port for the Haystack server & required \\
\hline root & path to the Haystack REST service & required \\
\hline authorization & the username and password for accessing the Haystack REST service & optional \\
\hline secure & whether to use HTTPS instead of HTTP & optional \\
\hline time zone & $\begin{array}{l}\text { time zone information: minutes offset from UTC, whether to use daylight savings } \\
\text { time, and the geographic location }\end{array}$ & required \\
\hline siteldentifier & identifier for the Records API server & required \\
\hline siteURI & URI for the Records API server metadata & required \\
\hline siteName & name of the Records API server & required \\
\hline siteTags & key-value pairs tagging the server with additional information & optional \\
\hline siteMeters & $\begin{array}{l}\text { list of meters to expose on the Records API server; the Haystack ID is followed by a } \\
\text { space and textual description. }\end{array}$ & required \\
\hline
\end{tabular}

\section{C++ Server and Client}

Both client and server applications have been implemented in $\mathrm{C}++$ for the Records API. See https://github.nrel.gov/d-star/cpp-records for details. There are GUI and command-line applications for serving comma-separated-value files and a GUI application for browsing Records API data sources.

\section{JavaScript Client Library and Web-Based Browser}

The client library for JavaScript relies on a few simple functions to interact with a Records API server. Full documentation for the JavaScript client library is available at http:/github.com/NREL/AESD/lib/javascript. The figure below shows the user interface of the general purpose Records API browser using this JavaScript library. 


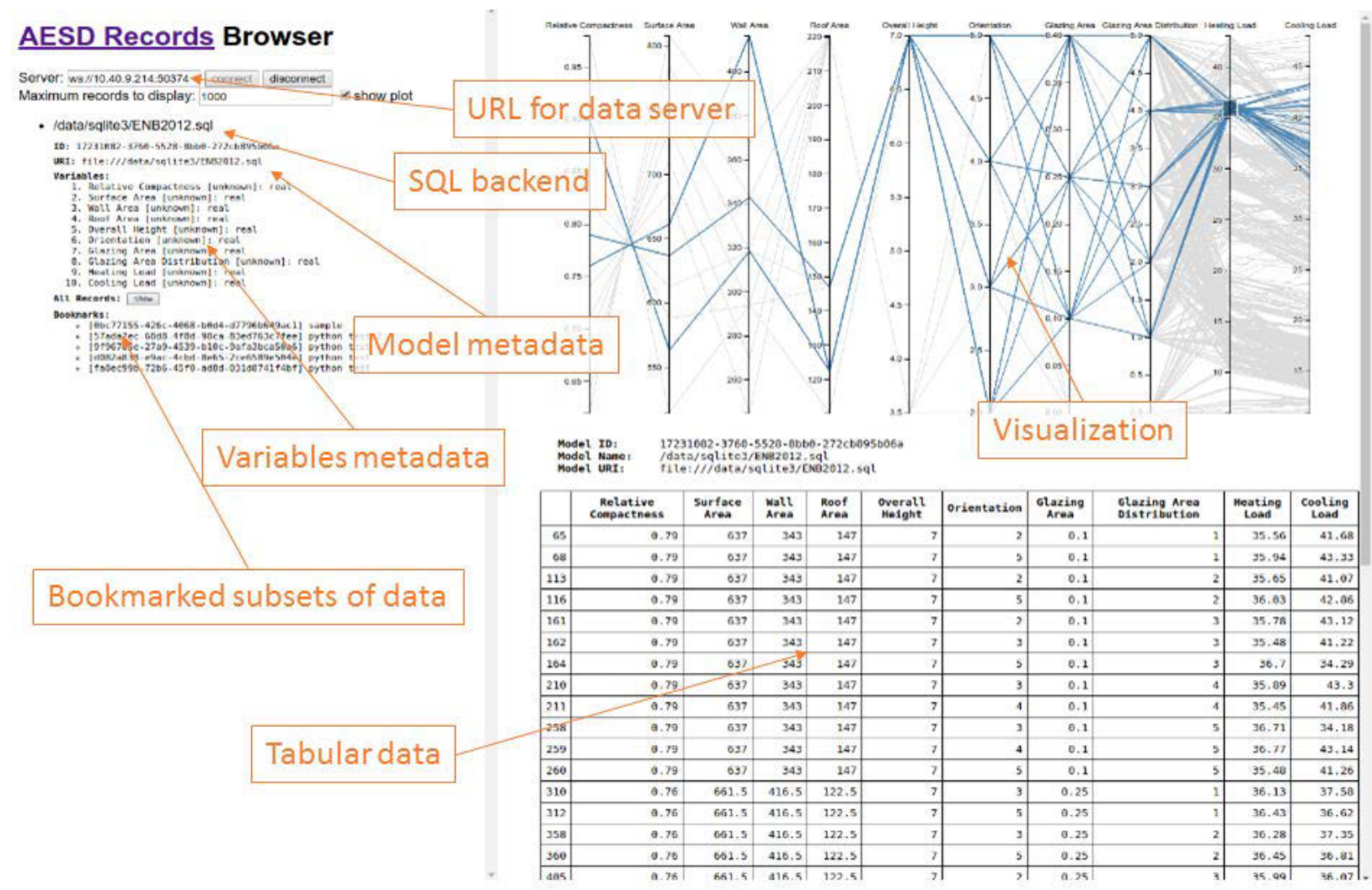

User interface for the Records API browser

Connect to a server connect (WSURL)

Here, wsURL is simply the URL of the server (e.g., ws://10.40.9.214:503761). This returns a connection object.

\section{Disconnect from a server disconnect(connection)}

Here, connection is the connection object returned by the connect function.

\section{Retrieve list of data models requestModelsMetadata(connection, modelId, notify, notifyError)}

Here, connection is the connection object returned by the connect function and modelId is either the string identifying the model or null if metadata for all models is requested. After all of the model metadata have been retrieved, the notify function is called with the list of model metadata objects as its argument; if an error occurs, notifyError is called with the error message as its argument. The function requestModel sMetadata returns a result object that contains a field done indicating whether all model metadata have been retrieved and a field models listing the model metadata retrieved so far. 


\section{Retrieve data records}

requestRecordsData(connection, modelId, maxRecords, variableIds, bookmarkId, notify, notifyError)

Here, connection is the connection object return by the connect function and modelId is the string identifying the model. After all of the data records have been retrieved, the notify function is called with the list of data records as its argument; if an error occurs, notifyError is called with the error message as its argument. The maxRecords argument specifies the maximum number of records to retrieve, variableIds may list the variables of interest, and bookmarkId restricts the results to bookmarked records. The function requestRecordsData returns a result object that contains a field done indicating whether all data records have been retrieved and a field data listing the data records retrieved so far.

\section{Retrieve list of bookmarks}

requestBookmarkMeta(connection, modelId, bookmarkId, notify, notifyError)

Here, connection is the connection object returned by the connect function, modelid is the string identifying the model, and bookmarkId is either the string identifying the bookmark or null if metadata for all bookmarks is requested. After all of the bookmark metadata have been retrieved, the notify function is called with the list of bookmark metadata as its argument; if an error occurs, notifyError is called with the error message as its argument. The function requestBookmarkMeta returns a result object that contains a field done indicating whether all bookmark metadata have been retrieved and a field bookmarks listing the bookmark metadata retrieved so far.

\section{Create/update a bookmark}

requestSaveBookmark(connection, modelId, name, filter, notify, notifyError)

Here, connection is the connection object returned by the connect function, modelId is the string identifying the model, and bookmarkId is either null for a new bookmark or the identifier for a bookmark being updated. The name field names the bookmark and the filter object describing the filtering operation for the bookmark. After the bookmark metadata has been created or updated, the notify function is called with the list of bookmark metadata as its argument; if an error occurs, notifyError is called with the error message as its argument. The function requestSaveBookmark returns a result object that contains a field done indicating whether all bookmark metadata have been retrieved and a field bookmarks listing the bookmark metadata retrieved so far.

\section{Python Client Library}

Full documentation for the Python client library is available at http://github.com/NREL/AESD/lib/python. 


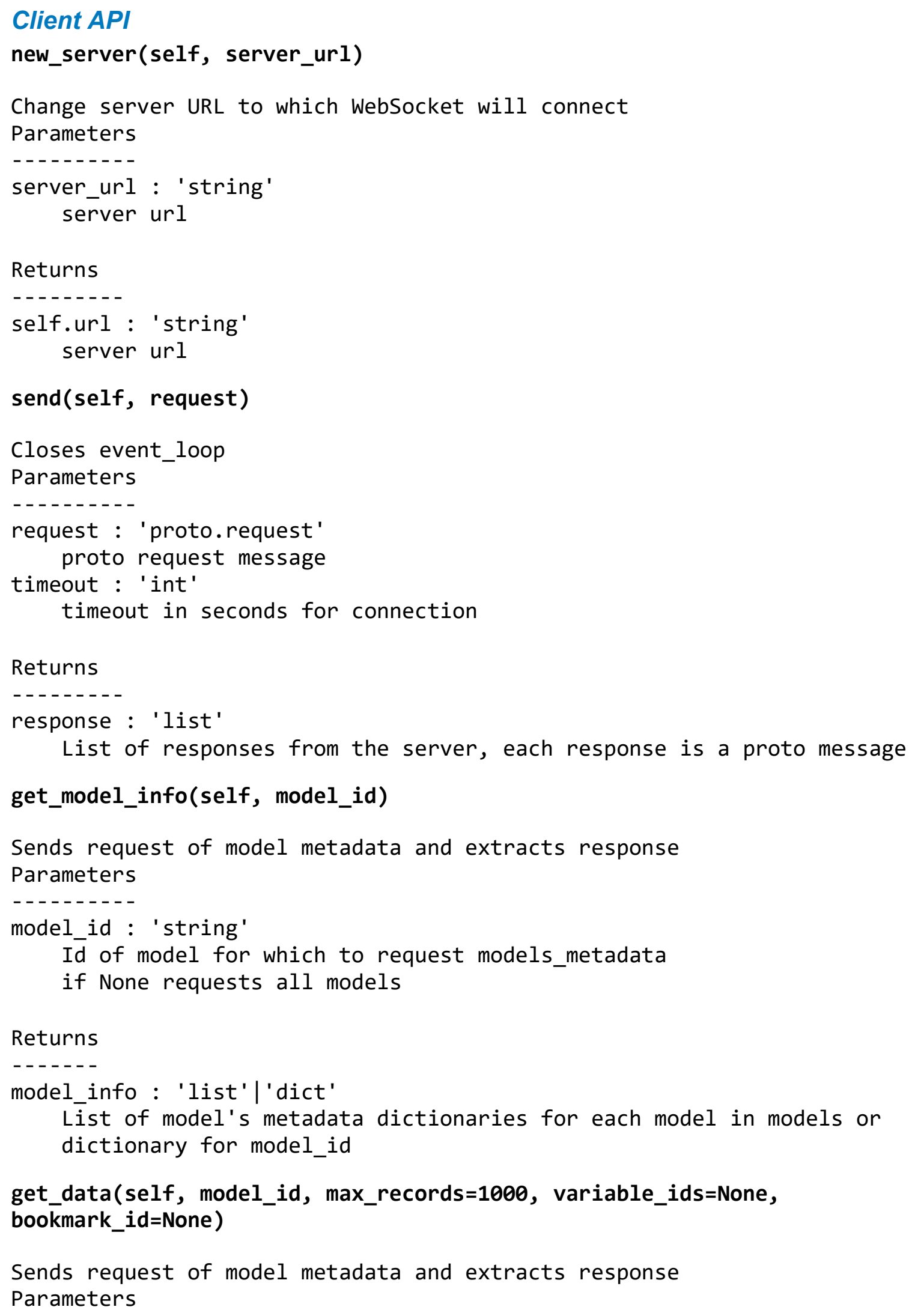


model_id : 'string'

Id of model for which to request records_data

max_records : 'int'

Number or records being request ( $\theta$ will return all records)

variable_ids : 'list'

List of variable ids (ints) to be requested

Will be returned in same order as request

Default=None, all variables will be returned (order?)

bookmark_id : 'int'

Request records_data based on bookmark id

\section{Returns}

- - - - -

data : 'pd.DataFrame'

Concatenated data from each response message

Variable ids replaced with names from model_info

do_work(self, model_id, inputs)

Sends request of model metadata and extracts response

Parameters

model_id : 'string'

Id of model for which to request records_data

inputs : 'dict'

Dictionary of $\{$ var_id: value $\}$ pairs

Returns

- - - - -

data : 'pd.DataFrame'

Concatenated data from each response message

Variable ids replaced with names from model_info

get_bookmark_info(self, model_id, bookmark_id)

Sends request of model metadata and extracts response

Parameters

--.--.-.-

model_id : 'string'

Id of model for which to request bookmark_meta bookmark_id : 'string'

Id of bookmark for which to request models_metadata

if None request all bookmarks

Returns

- - - - - -

model_info: 'list'|'dict'

List of model's metadata dictionaries for each model in models or

dictionary for model_id 
save_bookmark(self, model_id, name, content)

Sends request to save new bookmark

Parameters

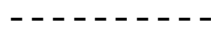

model_id : 'string'

Id of model for which to request bookmark_meta

name : 'string'

Name for new bookmark

content : 'list'|'tuple'

Contents of bookmark

list is a bookmark set

tuple is a bookmark interval

\section{Returns}

- - - - -

model_info : 'list'|'dict'

List of model's metadata dictionaries for each model in models or dictionary for model_id

\section{Example}

The figure below shows example usage of the Python Records API client.

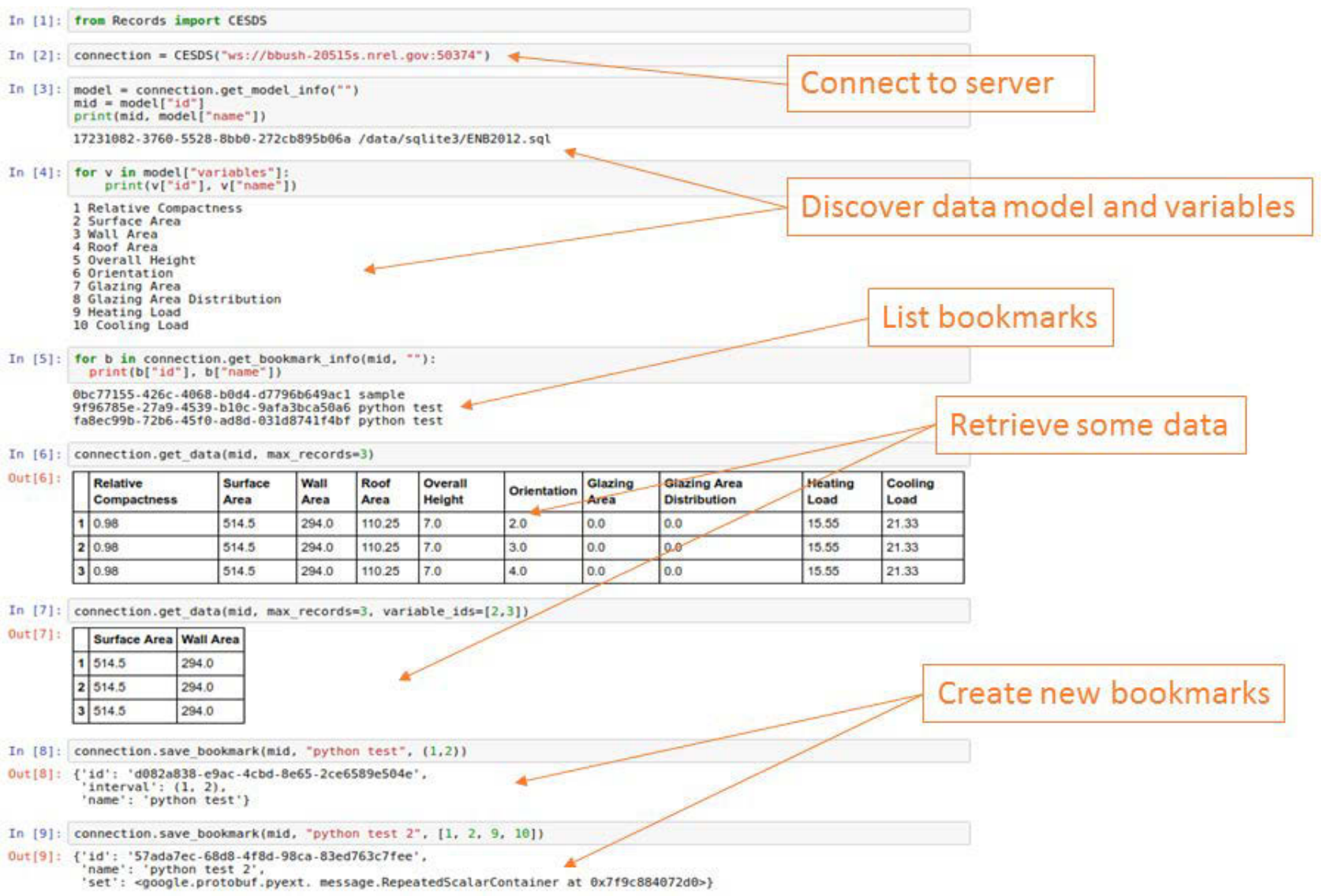

Example of a Python session using the Records API 


\section{Appendix}

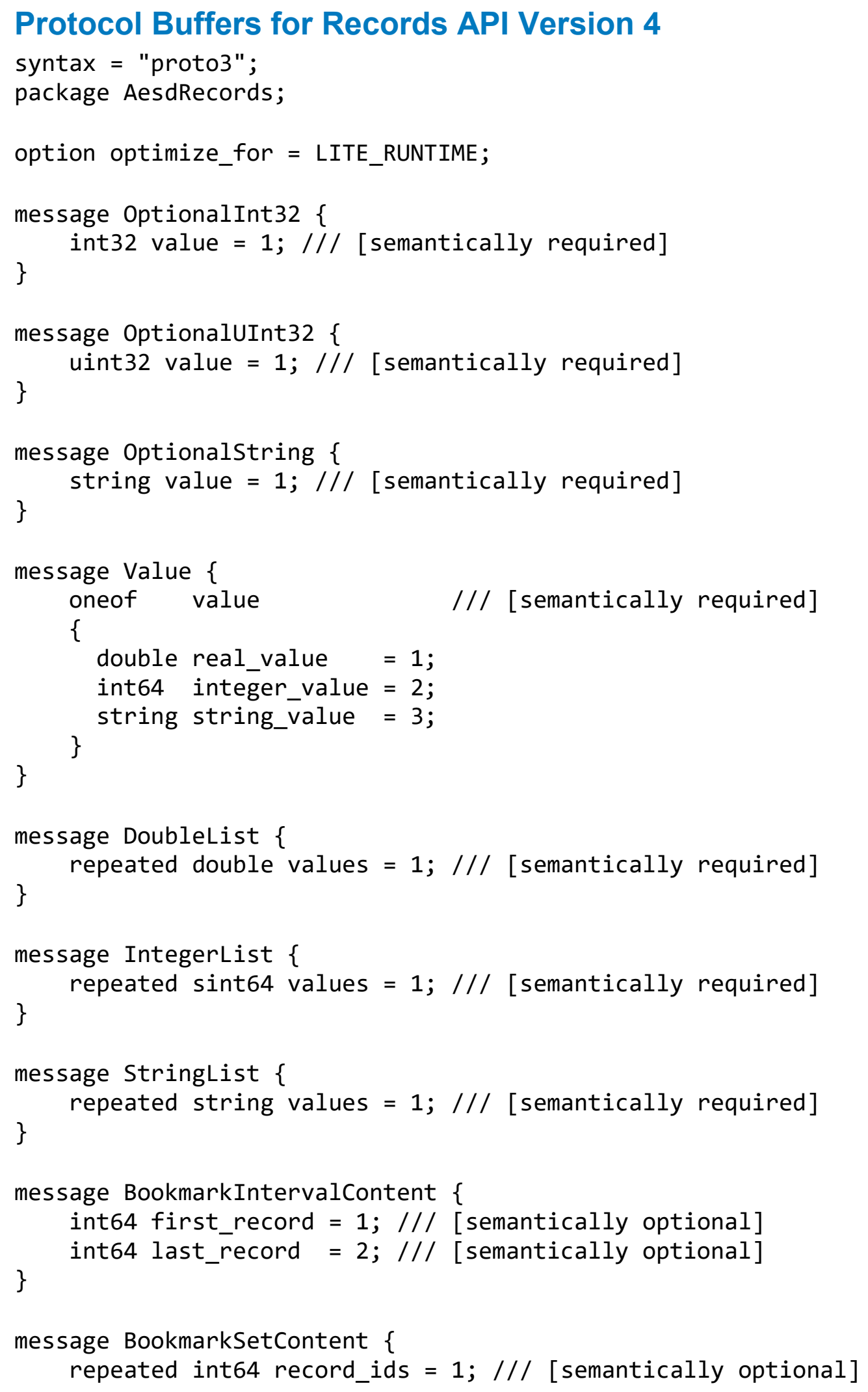




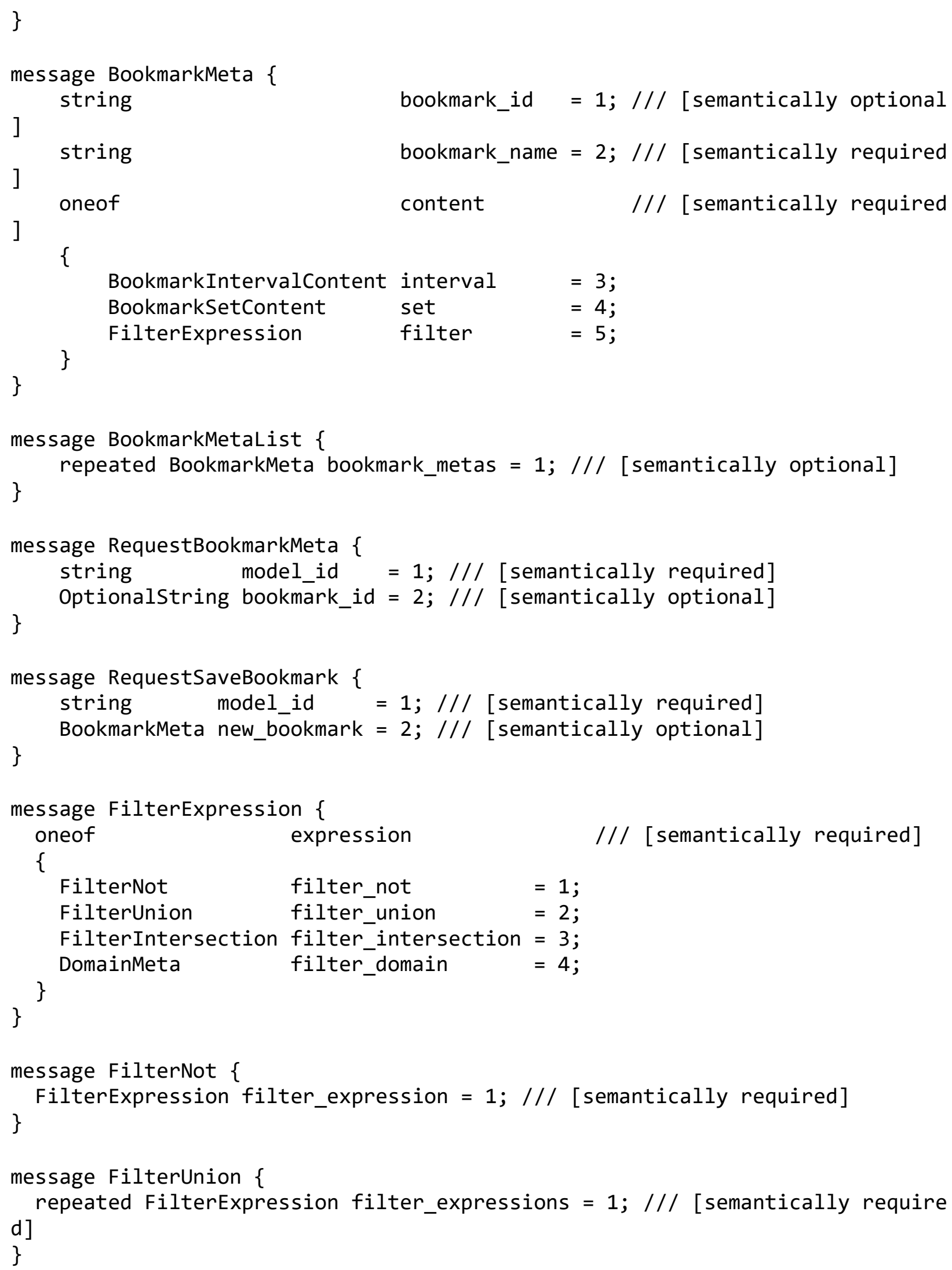




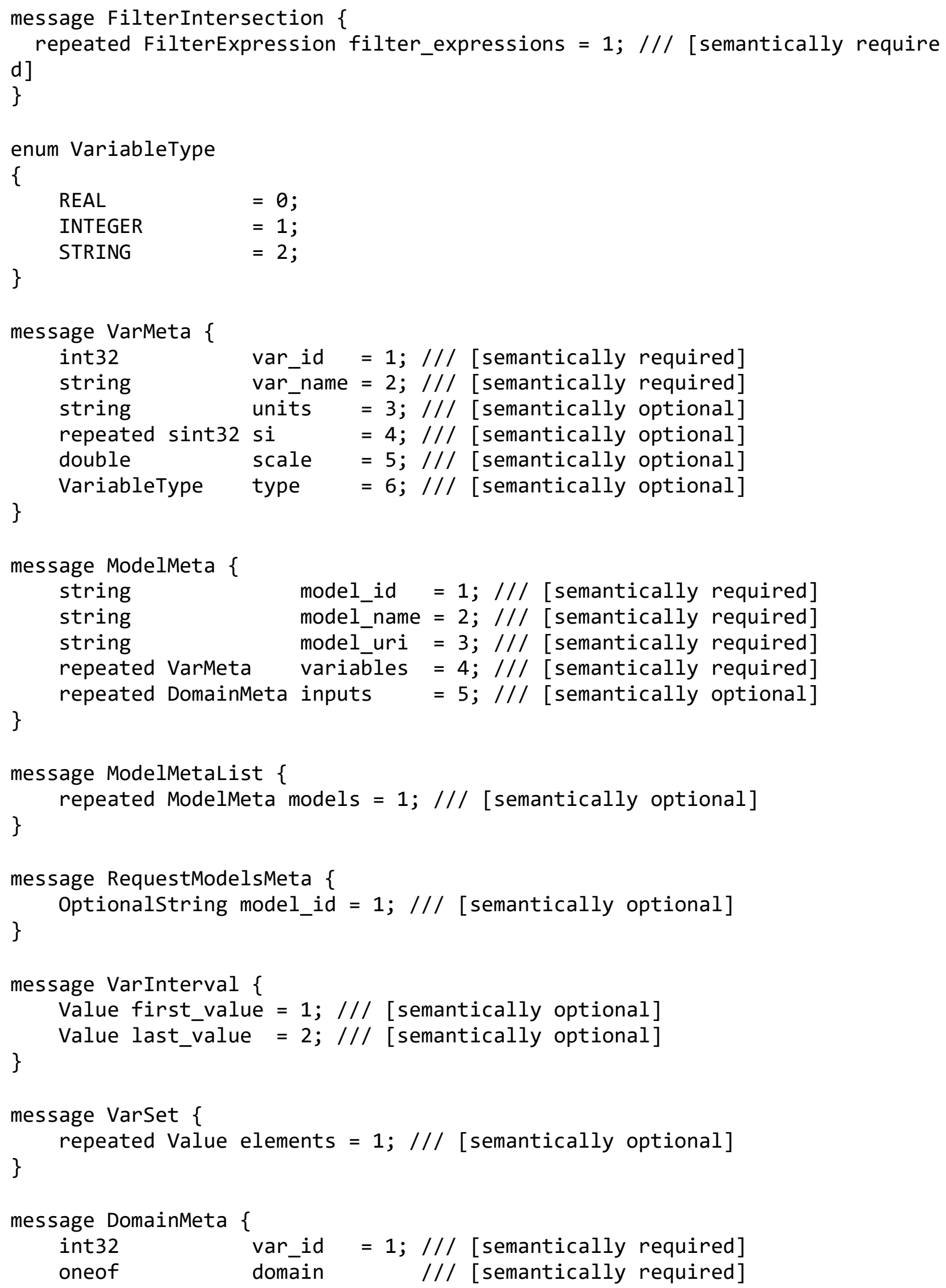




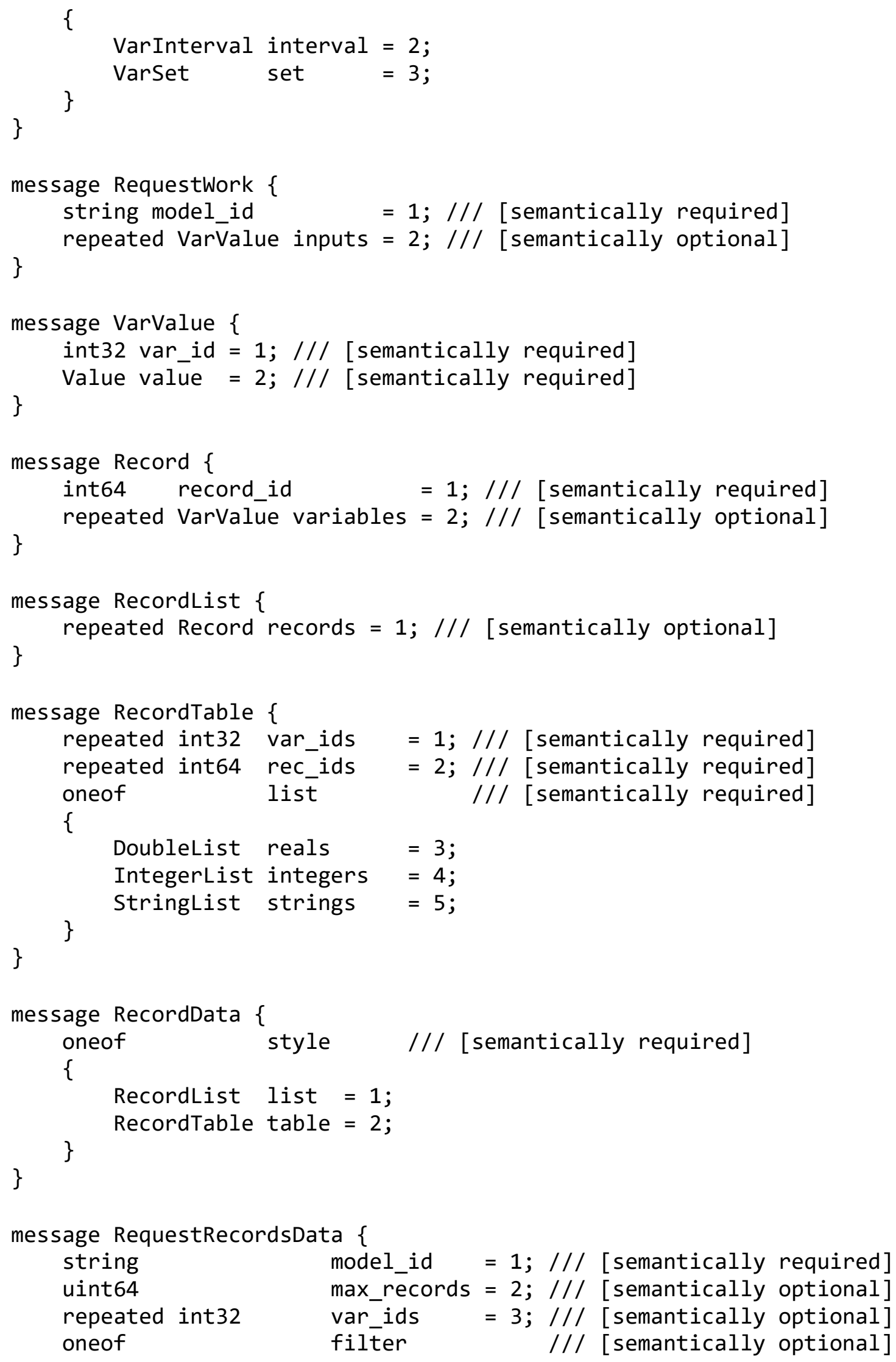




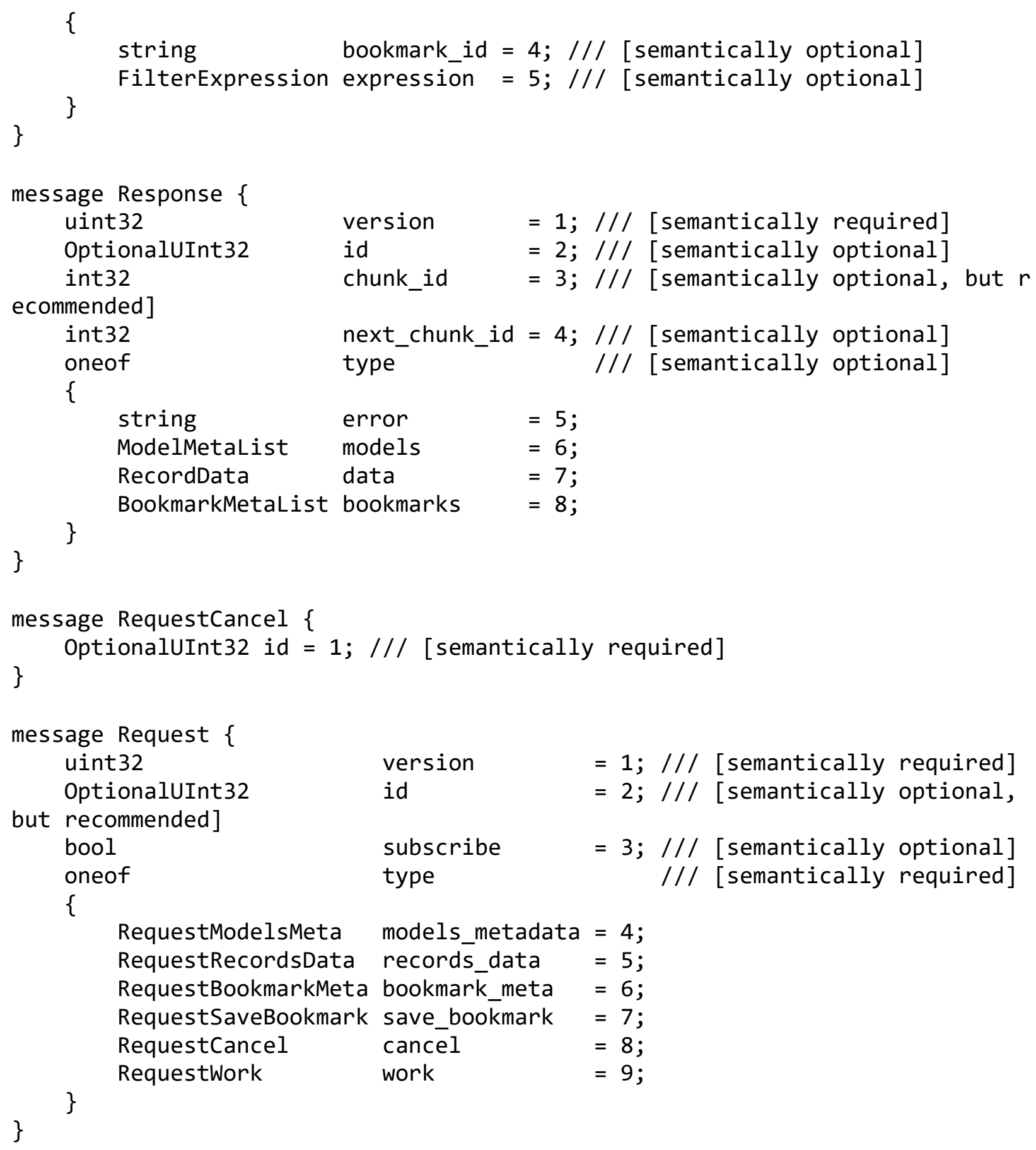




\section{References}

CoreOS. 2017a. “App Container Basics: Coreos.” Accessed September 6, 2017.

https://coreos.com/rkt/docs/latest/app-container.html.

CoreOS. 2017b. "Rkt Container Engine with Coreos.” Accessed September 6, 2017.

https://coreos.com/rkt.

Fowler, Martin. 2017. “UML Distilled.” Accessed April 11, 2017.

http://my.safaribooksonline.com/book/software-engineering-and-

development/uml/0321193687/sequence-diagrams/ch04.

Google Developers. 2017a. "Protocol Buffers | Google Developers.” Accessed April 11, 2017. https://developers.google.com/protocol-buffers/.

Google Developers. 2017b. "Protocol Buffers: Google's Data Interchange Format." Accessed April 11, 2017. https://github.com/google/protobuf/blob/master/README.md.

Internet Engineering Task Force. 2017. "RFC 6455 - The Websocket Protocol.” Accessed April 11, 2017. https://tools.ietf.org/html/rfc6455.

Oren Ben-Kiki, Clark Evans, Ingy döt Net. 2017. "YAML Specification Index." Accessed September 6, 2017. http://www.yaml.org/spec/.

Project Haystack. 2017. “Home - Project Haystack.” Accessed September 6, 2017. http://projecthaystack.org/. 